\title{
The Kolmogorov operator associated to a Burgers SPDE in spaces of continuous functions
}

\author{
Luigi Manca \\ Dipartimento di Matematica P. e A., \\ Università di Padova, \\ Via Trieste 63, \\ 35121 Padova, Italy \\ E-mail:manca@math.unipd.it
}

October 30, 2018

\begin{abstract}
We are concerned with a viscous Burgers equation forced by a perturbation of white noise type. We study the corresponding transition semigroup in a space of continuous functions weighted by a proper potential, and we show that the infinitesimal generator is the closure (with respect to a suitable topology) of the Kolmogorov operator associated to the stochastic equation. In the last part of the paper we use this result to solve the corresponding Fokker-Planck equation.

Key words: Burgers equation, white noise, Kolmogorov operator, transition semigroup, Fokker-Planck equation.
\end{abstract}

MSC 2000: 35Q53 (primary), 60H15, 35R15, 47D07 (secondary). 


\section{Introduction and preliminaries}

We consider the stochastic Burgers equation in the interval $[0,1]$ with Dirichlet boundary conditions perturbed by a space-time white noise

$$
\left\{\begin{array}{l}
d X=\left(D_{\xi}^{2} X+\frac{1}{2} D_{\xi}\left(X^{2}\right)\right) d t+d W, \quad \xi \in[0,1], t \geq 0 \\
X(t, 0)=X(t, 1)=0 \\
X(0, \xi)=x(\xi), \xi \in[0,1]
\end{array}\right.
$$

where $x \in L^{2}(0,1)$ and $W$ is a cylindrical Wiener process defined in a probability space $(\Omega, \mathcal{F}, \mathbb{P})$ and with values in $L^{2}(0,1)$.

The unknown $X$ is a real valued process depending on $\xi \in[0,1]$ and $t \geq 0$. It is known that there exists a unique solution with paths in $C\left([0, T], L^{2}(0,1)\right)$ (see [3]3,3]). In the paper [8][3,8] it has been proved that all the moments of the solution are finite. Starting by this result, we study the associated transition semigroup and the associated Kolmogorov operator in spaces of continuous functions with polynomial growth.

Let us write problem (11) in an abstract form. We denote by $L^{p}(0,1), p \geq$ 1 , the space of all real valued Lebesgue measurable functions $x:[0,1] \rightarrow \mathbb{R}$ such that

$$
|x|_{p}:=\left(\int_{0}^{1}|x(\xi)|^{p} d \xi\right)^{1 / p}<+\infty,
$$

and by $L^{\infty}(0,1)$ the space of all real valued Lebesgue measurable essentially bounded functions endowed with the usual supremum norm $|x|_{\infty}$, $x \in L^{\infty}(0,1)$. We consider the separable Hilbert space $H:=L^{2}(0,1)$ (norm $|\cdot|_{2}$ and inner product $\left.\langle x, y\rangle=|x y|_{1}, x, y \in H\right)$. As usual, $H^{k}(0,1), k \in \mathbb{N}$, is the Sobolev space of all functions in $H$ whose differentials belong to $H$ up to the order $k$, and $H_{0}^{1}(0,1)$ is the subspace of $H^{1}(0,1)$ of all functions whose trace at 0 and 1 vanishes. We define the unbounded self-adjoint operator $A$ in $H$ by

$$
A x=\frac{\partial^{2}}{\partial \xi^{2}} x, \quad x \in D(A)=H^{2}(0,1) \cap H_{0}^{1}(0,1) .
$$

Finally, we denote by $\left\{e_{k}\right\}_{k \in \mathbb{N}}$ the orthonormal system in $H$ given by the eigenvectors of $A$

$$
e_{k}(\xi)=\sqrt{\frac{2}{\pi}} \sin (k \xi), \quad \xi \in[0,1], k \in \mathbb{N}
$$


The cylindrical Wiener process $W(t)$ is given (formally) by

$$
W(t)=\sum_{k=1}^{\infty} \beta_{k}(t) e_{k}, \quad t \geq 0,
$$

where $\left\{\beta_{k}\right\}$ is a sequence of mutually independent standard Brownian motions on a stochastic basis $\left(\Omega, \mathcal{F},\left(\mathcal{F}_{t}\right)_{t \geq 0}, \mathbb{P}\right)$. We set

$$
b(x)=\frac{1}{2} D_{\xi}\left(x^{2}\right), \quad x \in D(b)=H_{0}^{1}(0,1) .
$$

Thanks to the introduced notations, we write problem (11) as

$$
\left\{\begin{array}{l}
d X=(A X+b(X)) d t+d W(t), \\
X(0)=x, \quad x \in H
\end{array}\right.
$$

The unique solution of (2) is denoted by $X(t, x)$. The corresponding transition semigroup $P_{t}, t \geq 0$ is given by

$$
P_{t} \varphi(x)=\mathbb{E}[\varphi(X(t, x))], \quad t \geq 0, \varphi \in \mathcal{C}_{b}(H), x \in H,
$$

where $\mathcal{C}_{b}(H)$ is the Banach space of all continuous and bounded real valued functions $\varphi: H \rightarrow \mathbb{R}$ endowed with the supremum norm

$$
\|\varphi\|_{0}=\sup _{x \in H}|\varphi(x)|
$$

and $\mathbb{E}$ means expectation. In [8],8] it has been proved the following

Proposition 1.1. For any $p \geq 2, k \geq 1, T>0$ there exists a constant $c_{p, k, T}$ such that

$$
\mathbb{E}\left[\sup _{t \in[0, T]}|X(t, x)|_{p}^{k}\right] \leq c_{p, k, T}\left(1+|x|_{p}^{k}\right) .
$$

Then the semigroup $P_{t}$ is extendible to spaces of real valued continuous functions with polynomial growth. In particular, $P_{t}$ acts on the space $\mathcal{C}_{b, V}\left(L^{6}(0,1)\right)$ of all continuous functions $\varphi: L^{6}(0,1) \rightarrow \mathbb{R}$ such that the function

$$
L^{6}(0,1) \rightarrow \mathbb{R}, \quad x \mapsto \frac{\varphi(x)}{1+V(x)}
$$

is bounded and where

$$
V(x):=|x|_{6}^{8}|x|_{4}^{2}, \quad x \in L^{6}(0,1) .
$$


The space $\mathcal{C}_{b, V}\left(L^{6}(0,1)\right)$, endowed with the norm

$$
\|\varphi\|_{0, V}:=\sup _{x \in L^{6}(0,1)} \frac{|\varphi(x)|}{1+V(x)}
$$

is a Banach space.

The semigroup $P_{t}$ is not strongly continuous in $\mathcal{C}_{b, V}\left(L^{6}(0,1)\right.$ ) (neither in $\mathcal{C}_{b}(H)$ ). However, it is strongly continuous with respect to weaker topologies. We follow here the approach of the $\pi$-convergence, suggested by Priola in [2424, 24]. The semigroup $P_{t}$ can be also studied in other frameworks, for instance with respect to uniform convergence on compact sets (see [22, 2], [16]6, 16] and Proposition 4.4 below). We define the infinitesimal generator of $P_{t}$ by setting

$$
\left\{\begin{array}{l}
D\left(K, \mathcal{C}_{b, V}\left(L^{6}(0,1)\right)\right)=\left\{\varphi \in \mathcal{C}_{b, V}\left(L^{6}(0,1)\right): \exists g \in \mathcal{C}_{b, V}\left(L^{6}(0,1)\right),\right. \\
\left.\lim _{t \rightarrow 0^{+}} \frac{P_{t} \varphi(x)-\varphi(x)}{t}=g(x), x \in L^{6}(0,1), \sup _{t \in(0,1)}\left\|\frac{P_{t} \varphi-\varphi}{t}\right\|_{0, V}<\infty\right\} \\
K \varphi(x)=\lim _{t \rightarrow 0^{+}} \frac{P_{t} \varphi(x)-\varphi(x)}{t}, \quad \varphi \in D\left(K, \mathcal{C}_{b, V}\left(L^{6}(0,1)\right)\right), x \in L^{6}(0,1) .
\end{array}\right.
$$

The Kolmogorov operator associated to equation (2) is formally given by

$$
K_{0} \varphi(x)=\frac{1}{2} \operatorname{Tr}\left[D^{2} \varphi(x)\right]+\langle x, A D \varphi(x)\rangle-\frac{1}{2}\left\langle D_{\xi} D \varphi(x), x^{2}\right\rangle,
$$

where $\varphi: L^{p}(0,1) \rightarrow \mathbb{R}$ is a suitable function and Tr means trace.

The main result of this paper consists in Theorem (7.1) below where we show that $\left(K, D\left(K, \mathcal{C}_{b, V}\left(L^{6}(0,1)\right)\right)\right)$ is the closure, with respect to the $\pi$-convergence, of the operator $K_{0}$ define on the domain $\mathcal{E}_{A}(H)$ (the set of exponential functions), which consists of the linear span of the real and imaginary part of the functions

$$
H \rightarrow \mathbb{C}, \quad x \mapsto e^{i\langle x, h\rangle} . \quad h \in D(A) .
$$

In other words, we show the set of exponential functions $\mathcal{E}_{A}(H)$ is a core for $\left(K, D\left(K, \mathcal{C}_{b, V}\left(L^{6}(0,1)\right)\right)\right)$, and $K \varphi=K_{0} \varphi, \forall \varphi \in \mathcal{E}_{A}(H)$.

Apart for the interest in having a better understanding of the operator $K_{0}$, the main motivation is to solve the corresponding Fokker-Planck equation

$$
\left\{\begin{array}{l}
\frac{d}{d t} \mu_{t}=K_{0}^{*} \mu_{t}, \quad t \geq 0 \\
\mu_{0}=\mu
\end{array}\right.
$$


where the family of measures $\mu_{t}, t \geq 0$ in the unknown and $\mu$ is a given Borel measure on $H$. The meaning of this problem will be explained by Theorem 1.2 below. Before introducing it, we need some notation.

If $E$ is a Banach space, $\mathcal{M}(E)$ is the set of all Borel finite measures on $E$. If $\mu \in \mathcal{M}(E)$, we denote by $|\mu|_{T V}$ the total variation measure of $\mu$. We shall denote by $\mathcal{M}_{V}\left(L^{6}(0,1)\right)$ the set of all $\mu \in \mathcal{M}\left(L^{6}(0,1)\right)$ such that

$$
\int_{L^{6}(0,1)} V(x)|\mu|_{T V}(d x)<\infty .
$$

The second main result of this paper is

Theorem 1.2. For any $\mu \in \mathcal{M}_{V}\left(L^{6}(0,1)\right)$ there exists an unique family of measures $\left\{\mu_{t}, t \geq 0\right\} \subset \mathcal{M}_{V}\left(L^{6}(0,1)\right)$ fulfilling

$$
\int_{0}^{T}\left(\int_{L^{6}(0,1)}(1+V(x))\left|\mu_{t}\right|_{T V}(d x)\right) d t<\infty, \quad \forall T>0
$$

and the Fokker-Planck equation

$$
\int_{L^{6}(0,1)} \varphi(x) \mu_{t}(d x)-\int_{L^{6}(0,1)} \varphi(x) \mu(d x)=\int_{0}^{t}\left(\int_{L^{6}(0,1)} K_{0} \varphi(x) \mu_{s}(d x)\right) d s,
$$

$t \geq 0, \varphi \in \mathcal{E}_{A}(H)$. Moreover, the solution is given by $P_{t}^{*} \mu, t \geq 0$.

The meaning of $P_{t}^{*} \mu$ will be made clear by Theorem 8.2 below.

In the papers [25|25, 25], [26|26, 26], the stochastic partial differential equation

$$
\left\{\begin{array}{l}
d X_{t}=(\Delta X(t)+F(X(t))) d t+\sqrt{Q} d W(t) \\
X(0)=x \in H
\end{array}\right.
$$

has been considered, where $H:=L^{2}(0,1), W(t), t \geq 0$ is a cylindrical Wiener process on $H, Q: H \rightarrow H$ is a nonnegative definite symmetric operator of trace class, $\Delta$ is the Dirichlet Laplacian on $(0,1), F: H_{0}^{1}(0,1) \rightarrow H$ is a measurable vector field of the form

$$
F(x)(r)=\frac{d}{d r}(\Psi \circ x)(r)+\Phi(r, x(r)), \quad x \in H_{0}^{1}(0,1), r \in(0,1) .
$$

The associated Kolmogorov operator is

$$
\mathcal{L} \varphi(x)=\frac{1}{2} \operatorname{Tr}\left(Q D^{2} \varphi(x)\right)+\langle\Delta x+F(x), D \varphi(x)\rangle,
$$


where $\varphi: H \rightarrow \mathbb{R}$ is a suitable cylindrical smooth function. Roughly speaking, the authors show that $\mathcal{L}$ can be extended to the generator of a strongly continuous semigroup in a space of weakly continuous functions weighted by a proper Lyapunov-type function. Then, they construct a Markov process which solves equation (8) in the sense of the martingale problem.

We stress that in [25|25, 25], 26126, 26] the noise is driven by a trace class operator, whereas in our case the perturbation is of white noise type. In this direction, the results of Theorem 8.2 seem to be new.

We mention also the papers [16]16, 16], [20[20, 20], [21|21, 21], where Kolmogorov operators of Ornstein-Uhlenbeck and reaction-diffusion type are considered in spaces of uniformly continuous functions.

In [88, 8] (see, also, [10|10, 10, [11]1, 11], [13113, 13], [14|14, 14], 99, 9], [19]19, 19]) the operator $K_{0}$ has been considered in the space $L^{2}(H ; \nu)$, where $\nu$ is the invariant measure (its existence is proved in [3], 3]) of the semigroup $P_{t}, t \geq 0$. In addition, several estimates are proved in order to ensure that the operator $K_{0}$ is $m$-dissipative in $L^{2}(H ; \nu)$. Therefore, the authors show that the operator $K_{0}$ can be uniquely extended to the infinitesimal generator of the semigroup $P_{t}$ in $L^{2}(H ; \nu)$.

The approach we use has been developed in [2020, 20], where a FokkerPlanck equation has been considered for Ornstein-Uhlenbeck operator perturbed by a Lipschitz and bounded term. In the paper [21|21, 21], this approach has been extended also to reaction-diffusion operators, and the Fokker-Planck equation has been solved for measure with finite moments up to a suitable degree.

We mention also [1818, 18], where Markov transition semigroups on spaces of measures have been considered, and the theory of such semigroups is developed.

Existence of measure valued solutions for equations involving second order partial differential operators in infinite dimensional spaces has been also considered in [11,1, However, in this paper we concentrate on uniqueness of the solution, whereas in [11,1] it has been shown existence results.

The paper is organized as follows: in the next two sections we shall introduce notation and we derive some preliminary results that will be used throghout the paper. In section 4 , we shall study the transition semigroup $P_{t}$ in in $\mathcal{C}_{b, V}\left(L^{6}(0,1)\right)$. In section 5 , we shall introduce the OrnsteinUhlenbeck semigroup and the corresponding operator in the spaces $\mathcal{C}_{b, 1}(H)$ and $\mathcal{C}_{b, V}\left(L^{6}(0,1)\right)$. Section 6 is devoted to study the Galerkin approximations of problems (11), (2) and to derive some fundamental estimate on the associated transition semigroup. In section 7 we show the main result of the 
paper, that is that the Kolmogorov operator $K_{0}$ is the characterization on a core of the operator (44). Finally, section 8 is devote to the proof of Theorem 8.2 .

\section{Notations and preliminary results}

If $E, E^{\prime}$ are, respectively, a topological space and a Banach space with norm $|\cdot|_{E^{\prime}}$, we denote by $\mathcal{C}_{b}\left(E, E^{\prime}\right)$ the Banach space of the bounded continuous function $\varphi: E \rightarrow E^{\prime}$ endowed with the supremum norm

$$
\|\varphi\|_{\mathcal{C}_{b}\left(E, E^{\prime}\right)}:=\sup _{x \in E}|\varphi(x)|_{E^{\prime}}
$$

When $E^{\prime}=\mathbb{R}$ we write $\mathcal{C}_{b}(E)=\mathcal{C}_{b}(E, \mathbb{R})$. If $E=H$, we simply denote by $\|\cdot\|_{0}$ the supremum norm of $\mathcal{C}_{b}(H)$. We also denote by $\mathcal{C}_{b, 1}(H)$ the Banach space of all continuous functions $f: H \rightarrow \mathbb{R}$ such that

$$
\|f\|_{0,1}:=\left\|\left(1+|\cdot|_{2}\right)^{-1} f\right\|_{0}<\infty .
$$

The set $\mathcal{C}_{b}^{1}(H)$ is the space of all $\varphi \in \mathcal{C}_{b}(H)$ which are Fréchet differentiable with continuous and bounded differential $D \varphi \in \mathcal{C}_{b}(H, H)$.

We now introduce the definition of $\pi$-convergence. Here, $E$ denote a topological space. A sequence $\left\{\varphi_{n}\right\}_{n \in \mathbb{N}} \subset \mathcal{C}_{b}(E)$ is said to be $\pi$-convergent to a function $\varphi \in \mathcal{C}_{b}(E)$ if

$$
\lim _{n \rightarrow \infty} \varphi_{n}(x)=\varphi(x), \quad \forall x \in E
$$

and

$$
\sup _{n \in \mathbb{N}}\left\|\varphi_{n}\right\|_{\mathcal{C}_{b}(E)}<\infty
$$

Similarly, the $m$-indexed sequence $\left\{\varphi_{n_{1}, \ldots, n_{m}}\right\}_{n_{1} \in \mathbb{N}, \ldots, n_{m} \in \mathbb{N}} \subset \mathcal{C}_{b}(E)$ is said to be $\pi$-convergent to $\varphi \in \mathcal{C}_{b}(E)$ if for any $i \in\{1, \ldots, m-1\}$ there exists an $i$-indexed sequence $\left\{\varphi_{n_{1}, \ldots, n_{i}}\right\}_{n_{1} \in \mathbb{N}, \ldots, n_{i} \in \mathbb{N}} \subset \mathcal{C}_{b}(E)$ such that

$$
\lim _{n_{i+1} \rightarrow \infty} \varphi_{n_{1}, \ldots, n_{i+1}} \stackrel{\pi}{=} \varphi_{n_{1}, \ldots, n_{i}}, \quad i \in\{1, \ldots, m-1\}
$$

and

$$
\lim _{n_{1} \rightarrow \infty} \varphi_{n_{1}} \stackrel{\pi}{=} \varphi
$$

We shall write

$$
\lim _{n_{1} \rightarrow \infty} \cdots \lim _{n_{m} \rightarrow \infty} \varphi_{n_{1}, \ldots, n_{m}} \stackrel{\pi}{=} \varphi
$$

or $\varphi_{n} \stackrel{\pi}{\rightarrow} \varphi$ as $n \rightarrow \infty$, when the sequence has one index. 
We recall that the operator $A$ is the infinitesimal generator of a strongly continuous semigroup in $H$, which we denote by $e^{t A}, t \geq 0$. Moreover, the semigroup $e^{t A}$ can be extended to $L^{p}(0,1)$, for any $p \geq 1$, and for all $p>1$ the constant $\lambda_{p}=2 p^{-1}(p-1) \pi^{2}$ verifies

$$
\left|e^{t A} x\right|_{p} \leq e^{\lambda_{p} t}|x|_{p}, \quad x \in L^{p}(0,1) .
$$

\section{Estimates on the solution}

Here we collect some properties of the solution of 1. A fundamental role will be played by the so-called stochastic convolution $W_{A}(t)$, which is formally given by

$$
W_{A}(t)=\int_{0}^{t} e^{(t-a) A} d W(s)=\sum_{k=1}^{\infty} \int_{0}^{t} e^{(t-a) A} e_{k} d \beta_{k}(s) .
$$

For any $t>0$, the process $W_{A}(t)$ has gaussian law of zero mean and covariance operator

$$
Q_{t} x=\int_{0}^{t} e^{2(t-a) A} x d s, \quad x \in H
$$

As easily seen, the operator $Q_{t}$ is trace class. Now set

$$
Y(t, x)=X(t, x)-W_{A}(t)
$$

We write (2) as

$$
\left\{\begin{array}{l}
Y(t, x)=e^{t A} x+\int_{0}^{t} e^{(t-s) A} \frac{\partial}{\partial \xi}\left(Y(s, x)+W_{A}(s, x)\right)^{2} d s \\
Y(0, x)=x, \quad x \in H
\end{array}\right.
$$

As we shall see, if $z(t) \in L^{p}(0,1)$ a.s., then $e^{t A} \frac{\partial}{\partial \xi} z^{2} \in L^{p}(0,1)$ is bounded. Then the above integral converges and the equation is meaningful. We now give the precise meaning of solution. We say that $X(t, x)$ is a mild solution of (2) if $Y(t, x)=X(t, x)-W_{A}(t)$ satisfies (10) for a.s. all $\omega \in \Omega$. The following result is proved in $[33,3]$.

Theorem 3.1. Let $x \in L^{p}(0,1), p \geq 2$. Then there exists a unique mild solution of equation (2), which belongs $\mathbb{P}$-a.s. to $C\left([0, T] ; L^{p}(0,1)\right)$, for any $T>0$. 
We prove uniform continuity with respect to the initial datum in a bounded neighborhood. In order to proceed, set

$$
\theta=\sup _{t \in[0, T]}\left|W_{A}(t)\right|_{\infty}, \quad T>0 .
$$

Clearly $\theta$ is a random variable, and $\theta<\infty$ a.s. We need the following estimates, proved in Lemma 3.1 of [3]3,3]

Lemma 3.2. For any $p \in[2, \infty)$ there exists $c_{p}>0$ such that if $Y(t, x)$ is a solution of (10), then

$$
|Y(t, x)|_{p} \leq c_{p}\left(\theta^{3}+|x|_{p}\right) e^{1+2 p \theta t} .
$$

We have the following

Theorem 3.3. For any $p \in[2, \infty)$ there exists a continuous function $c_{p}$ : $\left(\mathbb{R}^{+}\right)^{4} \rightarrow \mathbb{R}^{+}$such that

$$
|Y(t, x)-Y(t, y)|_{p} \leq c_{p}\left(t,|x|_{p},|y|_{p}, \theta\right)|x-y|_{p}, \quad x, y \in L^{p}(0,1)
$$

Proof. Here we follow [33, 3]. By (10) we have

$$
\begin{aligned}
& Y(t, x)-Y(t, y)=e^{t A}(x-y) \\
& \quad+\frac{1}{2} \int_{0}^{t} e^{(t-s) A} \frac{\partial}{\partial \xi}((Y(s, x)-Y(s, y))(Y(s, x)+Y(s, y)+2 W(s))) d s .
\end{aligned}
$$

then

$$
\begin{aligned}
& |Y(t, x)-Y(t, y)|_{p} \leq|x-y|_{p} \\
& +\frac{1}{2} \int_{0}^{t}\left|e^{(t-s) A} \frac{\partial}{\partial \xi}((Y(s, x)-Y(s, y))(Y(s, x)+Y(s, y)+2 W(s)))\right|_{p} d s .
\end{aligned}
$$

As well known, $e^{t A}, t \geq 0$ has smoothing properties. In particular, for any $s_{1}, s_{2} \in \mathbb{R}, s_{1} \leq s_{2}, r \geq 1, e^{t A}$ maps $W^{s_{1}, r}(0,1)$ into $W^{s_{2}, r}(0,1)$, for any $t>0$. Moreover, there exists $C_{1}>0$, depending on $s_{1}, s_{2}, r$, such that

$$
\left|e^{t A} z\right|_{W^{s_{2}, r}(0,1)} \leq C_{1}\left(1+t^{\frac{s_{1}-s_{2}}{2}}\right)|z|_{W^{s_{1}, r}(0,1)}, \quad z \in W^{s_{1}, r}(0,1),
$$

see Lemma 3, Part I in 2727,27. Using the Sobolev embedding theorem we have

$$
\left|e^{(t-s) A} \frac{\partial}{\partial \xi}((Y(s, x)-Y(s, y))(Y(s, x)+Y(s, y)+2 W(s)))\right|_{p}
$$




$$
\leq C_{1}\left|e^{(t-s) A} \frac{\partial}{\partial \xi}((Y(s, x)-Y(s, y))(Y(s, x)+Y(s, y)+2 W(s)))\right|_{W^{\frac{1}{p}, \frac{p}{2}}(0,1)}
$$

and, thanks to the above estimate with $s_{1}=-1, s_{2}=1 / p, r=p / 2$

$$
\begin{gathered}
\quad\left|e^{(t-s) A} \frac{\partial}{\partial \xi}((Y(s, x)-Y(s, y))(Y(s, x)+Y(s, y)+2 W(s)))\right|_{p} \\
\leq C_{1} C_{2}\left(1+(t-s)^{-\frac{1}{2}-\frac{1}{2 p}}\right) \\
\quad \times\left|\frac{\partial}{\partial \xi}((Y(s, x)-Y(s, y))(Y(s, x)+Y(s, y)+2 W(s)))\right|_{W^{-1, \frac{p}{2}}(0,1)} \\
\leq C_{1} C_{2}\left(1+(t-s)^{-\frac{1}{2}-\frac{1}{2 p}}\right)|(Y(s, x)-Y(s, y))(Y(s, x)+Y(s, y)+2 W(s))|_{\frac{p}{2}} \\
\leq C_{1} C_{2}\left(1+(t-s)^{-\frac{1}{2}-\frac{1}{2 p}}\right)|Y(s, x)-Y(s, y)|_{p}|Y(s, x)+Y(s, y)+2 W(s)|_{p} \\
\leq c_{p} C_{1} C_{2}\left(1+(t-s)^{-\frac{1}{2}-\frac{1}{2 p}}\right)|Y(s, x)-Y(s, y)|_{p} \\
\times\left(\left(2 \theta^{3}+|x|_{p}+|y|_{p}\right) e^{1+2 p \theta s}+2\right)
\end{gathered}
$$

Now the result follows by (12) and by Gronwall lemma (see, for instance, Lemma 7.1.1 in [17|17, 17]).

By recalling that $Y(t, x)=X(t, x)-W_{A}(t)$ it follows immediately the following result, which will be fundamental in the next section

Corollary 3.4. For any $p \in[2, \infty), x \in L^{p}(0,1), T>0$ we have, $\mathbb{P}$-a.s.,

$$
\lim _{\varepsilon \rightarrow 0^{+}} \sup _{|h|_{p}<\varepsilon}\left(\sup _{t \in[0, T]}|X(t, x+h)-X(t, x)|_{p}\right)=0
$$

\section{The transition semigroup in $\mathcal{C}_{b, V}\left(L^{6}(0,1)\right)$}

This section is devoted in studying the semigroup $P_{t}, t \geq 0$ in the space $\mathcal{C}_{b, V}\left(L^{6}(0,1)\right)$.

Remark 4.1. By Corollary 3.4 it follows that for any $\varphi \in \mathcal{C}_{b, V}\left(L^{6}(0,1)\right)$

$$
\lim _{\varepsilon \rightarrow 0} \sup _{|h|_{6}<\varepsilon, t \in[0, T]}\left|P_{t} \varphi(x+h)-P_{t} \varphi(x)\right|=0 .
$$

This, together with the estimate of Theorem 3.3, allows us to show that $P_{t}$ maps the space $\mathcal{C}_{b, V}\left(L^{6}(0,1)\right)$ into itself. 
Proposition 4.2. Formula (3) defines a semigroup of operators $\left(P_{t}\right)_{t \geq 0}$ in $\mathcal{C}_{b, V}\left(L^{6}(0,1)\right)$ and there exist two constants $c_{0} \geq 1, \omega_{0} \in \mathbb{R}$ and a family of probability measures $\left\{\pi_{t}(x, \cdot), t \geq 0, x \in L^{6}(0,1)\right\} \subset \mathcal{M}_{V}\left(L^{6}(0,1)\right)$ such that

(i) $P_{t} \in \mathcal{L}\left(\mathcal{C}_{b, V}\left(L^{6}(0,1)\right)\right)$ and $\left\|P_{t}\right\|_{\mathcal{L}\left(\mathcal{C}_{b, V}\left(L^{6}(0,1)\right)\right.} \leq c_{0} e^{\omega_{0} t}$;

(ii) $P_{t} \varphi(x)=\int_{H} \varphi(y) \pi_{t}(x, d y)$, for any $t \geq 0, \varphi \in \mathcal{C}_{b, V}\left(L^{6}(0,1)\right), x \in$ $L^{6}(0,1)$;

(iii) for any $\varphi \in \mathcal{C}_{b, V}\left(L^{6}(0,1)\right), x \in L^{6}(0,1)$, the function $\mathbb{R}^{+} \rightarrow \mathbb{R}, t \mapsto$ $P_{t} \varphi(x)$ is continuous.

(iv) $P_{t} P_{s}=P_{t+s}$, for any $t, s \geq 0$ and $P_{0}=I$;

(v) for any $\varphi \in \mathcal{C}_{b, V}\left(L^{6}(0,1)\right)$ and any sequence $\left(\varphi_{n}\right)_{n \in \mathbb{N}} \subset \mathcal{C}_{b, V}\left(L^{6}(0,1)\right)$ such that

$$
\lim _{n \rightarrow \infty} \frac{\varphi_{n}}{1+V} \stackrel{\pi}{=} \frac{\varphi}{1+V}
$$

we have, for any $t \geq 0$,

$$
\lim _{n \rightarrow \infty} \frac{P_{t} \varphi_{n}}{1+V} \stackrel{\pi}{=} \frac{P_{t} \varphi}{1+V}
$$

Proof. (i). Take $\varphi \in \mathcal{C}_{b, V}\left(L^{6}(0,1)\right), t>0$. We have to show that $P_{t} \varphi \in$ $\mathcal{C}_{b, V}\left(L^{6}(0,1)\right)$. By Proposition 1.1 it follows that

$$
\left|P_{t} \varphi(x)\right| \leq\|\varphi\|_{0, V}(1+\mathbb{E}[V(X(t, x))]) \leq c\|\varphi\|_{0, V}(1+V(x)),
$$

for some $c>0$. Then, we have to show that the function $L^{6}(0,1) \rightarrow \mathbb{R}$. $x \mapsto P_{t} \varphi(x)$ is continuous. Fix $x_{0} \in L^{6}(0,1)$. We have

$$
\left|P_{t} \varphi\left(x_{0}+h\right)-P_{t} \varphi\left(x_{0}\right)\right| \leq \mathbb{E}\left[\varphi\left(X\left(t, x_{0}+h\right)\right)-\varphi\left(X\left(t, x_{0}\right)\right) \mid\right] .
$$

By Corollary 3.4 we have that $\left|X\left(t, x_{0}+h\right)-X\left(t, x_{0}\right)\right|_{6} \rightarrow 0 \mathbb{P}$-a.s. as $|h|_{6} \rightarrow 0$. Then, by the continuity of $\varphi$ it follows $\left|\varphi\left(X\left(t, x_{0}+h\right)\right)-\varphi\left(X\left(t, x_{0}\right)\right)\right| \rightarrow$ $0 \mathbb{P}$-a.s. as $|h|_{6} \rightarrow 0$. On the other hand, $\varphi\left(X\left(t, x_{0}+h\right)\right)$ has bounded expectation, uniformly in any $L^{6}(0,1)$-ball of center $x_{0}$. Then, it follows that $P_{t} \varphi\left(x_{0}+h\right) \rightarrow P_{t} \varphi\left(x_{0}\right)$ has $|h|_{6} \rightarrow 0$. (i) is proved.

(ii). Take $\varphi \in \mathcal{C}_{b, V}\left(L^{6}(0,1)\right)$, and consider a sequence $\left(\varphi_{n}\right)_{n \in \mathbb{N}} \subset \mathcal{C}_{b}\left(L^{6}(0,1)\right)$ such that

$$
\lim _{n \rightarrow \infty} \frac{\varphi_{n}}{1+V} \stackrel{\pi}{=} \frac{\varphi}{1+V}
$$


Since $\pi_{t}(t, \cdot)$ is the image measure of $X(t, x)$ in $H$, the representation (ii) holds for any $\varphi_{n}$, that is

$$
P_{t} \varphi_{n}(x)=\mathbb{E}\left[\varphi_{n}(X(t, x))\right]=\int_{H} \varphi_{n}(y) \pi_{t}(x, d y), \quad x \in H .
$$

By Proposition 1.1 we can apply the dominated convergence theorem to find the result.

(iii). For any $\varphi \in \mathcal{C}_{b, V}\left(L^{6}(0,1)\right), x \in L^{6}(0,1), t, s \geq 0$ we have

$$
P_{t} \varphi(x)-P_{s} \varphi(x)=\mathbb{E}[\varphi(X(t, x))-\varphi(X(s, x))]
$$

Since for any $T>0$ we have $X(\cdot, x) \in C\left([0, T], L^{6}(0,1)\right) \mathbb{P}$-a.s. (cfr. Theorem 3.1), (iii) follows.

(iv). Take $\varphi \in \mathcal{C}_{b, V}\left(L^{6}(0,1)\right)$ and consider a sequence $\left(\varphi_{n}\right)_{n \in \mathbb{N}} \subset \mathcal{C}_{b}\left(L^{6}(0,1)\right)$ such that $(1+V)^{-1} \varphi_{n} \stackrel{\pi}{\rightarrow}(1+V)^{-1} \varphi$ as $n \rightarrow \infty$. By the markovianity of the process $X(t, x)$ it follows that (iv) holds true for any $\varphi_{n}$. Then, since by (iii) $(1+V)^{-1} P_{t} \varphi_{n} \stackrel{\pi}{\rightarrow}(1+V)^{-1} P_{t} \varphi$ as $n \rightarrow \infty$, still by (iii) we find

$$
\frac{P_{t+s} \varphi}{1+V} \stackrel{\pi}{=} \lim _{n \rightarrow \infty} \frac{P_{t+s} \varphi_{n}}{1+V}=\lim _{n \rightarrow \infty} \frac{P_{t} P_{s} \varphi_{n}}{1+V} \stackrel{\pi}{=} \frac{P_{t} P_{s} \varphi}{1+V} .
$$

(v). Since (ii) holds and $\pi_{t}(x, d y) \in \mathcal{M}_{V}\left(L^{6}(0,1)\right)$, the result follows by the dominated convergence Theorem. This concludes the proof.

Proposition 4.3. Let $X(t, x)$ be the mild solution of problem (21) and let $P_{t}$, $t \geq 0$ be the associated transition semigroups in the space $\mathcal{C}_{b, V}\left(L^{6}(0,1)\right)$ defined by (3). Let also $\left(K, D\left(K, \mathcal{C}_{b, V}\left(L^{6}(0,1)\right)\right)\right)$ be the associated infinitesimal generators, defined by (4). Then

(i) for any $\varphi \in D\left(K, \mathcal{C}_{b, V}\left(L^{6}(0,1)\right)\right)$, we have $P_{t} \varphi \in D\left(K, \mathcal{C}_{b, V}\left(L^{6}(0,1)\right)\right)$ and $K P_{t} \varphi=P_{t} K \varphi, t \geq 0$

(ii) for any $\varphi \in D\left(K, \mathcal{C}_{b, V}\left(L^{6}(0,1)\right)\right), x \in H$, the map $[0, \infty) \rightarrow \mathbb{R}, t \mapsto$ $P_{t} \varphi(x)$ is continuously differentiable and $(d / d t) P_{t} \varphi(x)=P_{t} K \varphi(x)$;

(iii) for any $\varphi \in \mathcal{C}_{b, V}\left(L^{6}(0,1)\right)$, $t>0$, the function

$$
H \rightarrow \mathbb{R}, \quad x \mapsto \int_{0}^{t} P_{s} \varphi(x) d s
$$

belongs to $D\left(K, \mathcal{C}_{b, V}\left(L^{6}(0,1)\right)\right)$, and it holds

$$
K\left(\int_{0}^{t} P_{s} \varphi d s\right)=P_{t} \varphi-\varphi
$$


(iv) for any $\lambda>\omega_{0}$, where $\omega_{0}$ is as in Proposition 4.2. the linear operator $R(\lambda, K)$ on $\mathcal{C}_{b, V}\left(L^{6}(0,1)\right)$ defined by

$$
R(\lambda, K) f(x)=\int_{0}^{\infty} e^{-\lambda t} P_{t} f(x) d t, \quad f \in \mathcal{C}_{b, V}\left(L^{6}(0,1)\right), x \in L^{6}(0,1)
$$

satisfies, for any $f \in \mathcal{C}_{b, V}\left(L^{6}(0,1)\right)$

$$
\begin{gathered}
R(\lambda, K) \in \mathcal{L}\left(\mathcal{C}_{b, V}\left(L^{6}(0,1)\right)\right), \quad\|R(\lambda, K)\|_{\mathcal{L}\left(\mathcal{C}_{b, V}\left(L^{6}(0,1)\right)\right)} \leq \frac{c_{0}}{\lambda-\omega_{0}} \\
R(\lambda, K) f \in D\left(K, \mathcal{C}_{b, V}\left(L^{6}(0,1)\right)\right), \quad(\lambda I-K) R(\lambda, K) f=f,
\end{gathered}
$$

where $c_{0}$ is as in Proposition 4.2. We call $R(\lambda, K)$ the resolvent of $K$ at $\lambda$.

Proof. (i), (ii) are an easy consequence of (4) and Proposition 4.2 (see the proof of Proposition 2.8 in $[20 \mid 20,20]$ ).

Let us show (iii). First, we have to check that $\int_{0}^{t} P_{s} f d s$ belongs to $\mathcal{C}_{b, V}\left(L^{6}(0,1)\right)$. By (i) of Proposition 4.2, for any $x \in L^{6}(0,1)$ we have

$$
\left|\int_{0}^{t} P_{s} \varphi(x) d s\right| \leq\|\varphi\|_{0, V} c_{0} \int_{0}^{t} e^{\omega_{0} s} d s(1+V(x)) .
$$

then,

$$
\sup _{x \in L^{6}(0,1)} \frac{1}{1+V(x)}\left|\int_{0}^{t} P_{s} \varphi(x) d s\right|<\infty .
$$

Now let us fix $\varepsilon>0, x_{0} \in L^{6}(0,1)$ and take $\delta>0$ such that

$$
\sup _{s \in[0, t]} \sup _{\substack{h \in L^{6}(0,1) \\|h|_{6}<\delta}}\left|P_{s} \varphi\left(x_{0}+h\right)-P_{s} \varphi\left(x_{0}\right)\right|<\frac{\varepsilon}{t} .
$$

The constant $\delta>0$ exists thank to Remark 4.1. Therefore, for any $h \in$ $L^{6}(0,1),|h|_{6}<\delta$ we have

$$
\left|\int_{0}^{t} P_{s} \varphi\left(x_{0}+h\right) d s-\int_{0}^{t} P_{s} \varphi\left(x_{0}\right) d s\right| \leq \int_{0}^{t}\left|P_{s} \varphi\left(x_{0}+h\right)-P_{s} \varphi\left(x_{0}\right)\right| d s<\varepsilon .
$$

By the arbitrariness of $x_{0}$, it follows $\int_{0}^{t} P_{s} \varphi d s \in \mathcal{C}_{b, V}\left(L^{6}(0,1)\right)$. The rest of the proof is essentially the same done for Theorem 2.9 in [20120, 20]. 


\subsection{Further results}

In this section we show that the semigroup $P_{t}$ is strongly continuous with respect to the weak convergence which has been introduced in [22, 2].

Proposition 4.4. For any $p \in[2, \infty)$ and any compact set $K \subset L^{p}(0,1)$ it holds

$$
\lim _{t \rightarrow 0^{+}} \sup _{x \in K}|X(t, x)-x|_{p}=0 \quad \mathbb{P}-\text { a.s. }
$$

Proof. Fix $T>0$. As usual, $\theta$ is the random variable in (11). For any $x \in L^{p}(0,1), 0<t<T$ we have

$$
\begin{gathered}
|X(t, x)-x|_{p} \leq|Y(t, x)-x|_{p}+\left|W_{A}(t)\right|_{p} \\
\leq\left|e^{t A} x-x\right|_{p}+\left|W_{A}(t)\right|_{p}+\int_{0}^{t}\left|e^{(t-s) A} \frac{\partial}{\partial \xi}\left((Y(s, x)-W(s))^{2}\right)\right|_{p} d s
\end{gathered}
$$

By arguing as in the proof of Theorem 3.3 we find

$$
\begin{gathered}
\int_{0}^{t}\left|e^{(t-s) A} \frac{\partial}{\partial \xi}\left((Y(s, x)-W(s))^{2}\right)\right|_{p} d s \\
\left.\leq C_{1} C_{2} \int_{0}^{t}\left(1+(t-s)^{-\frac{1}{2}-\frac{1}{2 p}}\right) \mid Y(s, x)-W(s)\right)\left.\right|_{p} d s
\end{gathered}
$$

for some $C_{1}, C_{2}>0$. Thanks to Lemma 3.2, the last term in the right-hand side is bounded by

$$
C_{1} C_{2} \int_{0}^{t}\left(1+(t-s)^{-\frac{1}{2}-\frac{1}{2 p}}\right)\left(c_{p}\left(\theta^{3}+|x|_{p}\right) e^{1+2 p \theta s}+\theta\right) d s
$$

Finally, we have found

$$
\begin{gathered}
|X(t, x)-x|_{p} \leq\left|e^{t A} x-x\right|_{p}+\left|W_{A}(t)\right|_{p} \\
+C_{1} C_{2} \int_{0}^{t}\left(1+(t-s)^{-\frac{1}{2}-\frac{1}{2 p}}\right)\left(c_{p}\left(\theta^{3}+|x|_{p}\right) e^{1+2 p \theta s}+\theta\right) d s .
\end{gathered}
$$

According to the fact that $\left|e^{t A} x-x\right|_{p} \rightarrow 0$ as $t \rightarrow 0^{+}$, uniformly on compact sets of $L^{p}(0,1)$, and that $|W(t)|_{p} \rightarrow 0 \mathbb{P}$-a.s. as $t \rightarrow 0^{+}$we get the result.

Thanks to Proposition 4.4 we are able to improve the previous result. We have 
Proposition 4.5. The semigroup $P_{t}, t \geq 0$ is a strongly continuous semigroup in the mixed topology of $\mathcal{C}_{b, V}\left(L^{6}(0,1)\right)$. That is for any $\varphi \in \mathcal{C}_{b, V}\left(L^{6}(0,1)\right)$, $T>0$ and any compact set $K \subset L^{6}(0,1)$ we have

$$
\left.\lim _{t \rightarrow 0^{+}} \sup _{x \in K} \mid P_{t} \varphi(x)-\varphi(x)\right) \mid=0
$$

and

$$
\sup _{t \in[0, T]}\left\|P_{t} \varphi\right\|_{0, V}<\infty
$$

\section{The Ornstein-Uhlenbeck operator}

Here we consider the transition semigroup associated to the mild solution of the linear stochastic equation

$$
\left\{\begin{array}{l}
d Z(t, x)=A Z(t, x) d t+d W(t), \quad t \geq 0 \\
Z(0, x)=x \in H
\end{array}\right.
$$

We recall that the solution is given by the process

$$
Z(t, x)=e^{t A} x+W_{A}(t),
$$

where $W_{A}(t), t \geq 0$ is the stochastic convolution introduced in (9). We recall that the process $Z(t, x)$ has a version which is, a.s. for $\omega \in \Omega, \alpha$-Hölder continuous with respect to $(t, x)$, for any $\alpha \in\left(0, \frac{1}{4}\right)$ (see [10|10,10], Theorem 5.20 and Example 5.21). For any $t \geq 0$ we define the Ornstein-Uhlenbeck (OU) semigroup $R_{t}, t \geq 0$ by

$$
R_{t} \varphi(x)=\mathbb{E}[\varphi(Z(t, x))], \quad t \geq 0, \varphi \in \mathcal{C}_{b}(H), x \in H
$$

where $Z(t, x)$ is the mild solution of (14). It is well known (see, for instance, [44, 4]) the following result

Proposition 5.1. For any $p, k \geq 1, T>0$ there exists a constant $c_{p, k, T}>0$ such that

$$
\mathbb{E}\left[\sup _{t \in[0, T]}|Z(t, x)|_{p}^{k}\right] \leq c_{p, k, T}\left(1+|x|_{p}^{k}\right) .
$$

This easily implies that $R_{t}, t \geq 0$ can be extended to a semigroup in the space $\mathcal{C}_{b, 1}(H)$ (see also [2]2,2], [16]16, 16]).In this space, $R_{t}$ is not strongly continuous with respect to the supremum norm. However, it is well known that it is strongly continuous with respect to the so called mixed topology 
(see [16]16, 16]) or, equivalently (see [16]16, 16] for this result), with respect to the weakly convergence introduce by Cerrai in [2], 2]. We recall that a sequence $\left\{\varphi_{n}\right\}_{n} \subset \mathcal{C}_{b, 1}(H)$ is said to be weakly convergent to $\varphi \in \mathcal{C}_{b, 1}(H)$ if $\varphi_{n}(x) \rightarrow \varphi(x), \forall x \in H$, as $n \rightarrow \infty$ uniformly on compact sets of $H$ and $\sup _{n}\left\|\varphi_{n}\right\|_{0,1}<\infty$. So, following [2]2,2], it is possible to define an infinitesimal operator

$$
L: D\left(L, \mathcal{C}_{b, 1}(H)\right) \subset \mathcal{C}_{b, 1}(H) \rightarrow \mathcal{C}_{b, 1}(H)
$$

through its resolvent operator (see Definition 3.3 and Remark 3.4 in [22,2]).

By Theorem 3.7 of [2424,24], it follows that the operator $\left(L, D\left(L, \mathcal{C}_{b, 1}(H)\right)\right)$ coincides with the operator

$$
\left\{\begin{array}{l}
D\left(L,\left(L, \mathcal{C}_{b, 1}(H)\right)\right)=\left\{\varphi \in \mathcal{C}_{b, 1}(H): \exists g \in \mathcal{C}_{b, 1}(H),\right. \\
\left.\quad \lim _{t \rightarrow 0^{+}} \frac{R_{t} \varphi(x)-\varphi(x)}{t}=g(x), \forall x \in H, \sup _{t \in(0,1)}\left\|\frac{R_{t} \varphi-\varphi}{t}\right\|_{0,1}<\infty\right\} \\
L \varphi(x)=\lim _{t \rightarrow 0^{+}} \frac{R_{t} \varphi(x)-\varphi(x)}{t}, \quad \varphi \in D\left(L, \mathcal{C}_{b, 1}(H)\right), x \in H .
\end{array}\right.
$$

It is well known the following fact (see [10|10, 10, [13/13, 13])

$$
R_{t} e^{i\langle\cdot h\rangle}(x)=e^{i\left\langle e^{t A} x, h\right\rangle-\frac{1}{2}\left\langle Q_{t} h, h\right\rangle}, \quad t \geq 0, x, h \in H .
$$

This implies that $R_{t}: \mathcal{E}_{A}(H) \rightarrow \mathcal{E}_{A}(H), \forall t \geq 0$ or, in other words, that the set $\mathcal{E}_{A}(H)$ is stable for $R_{t}$. We denote by $L_{0}$ is the Ornstein-Uhlenbeck operator

$$
L_{0} \varphi(x)=\frac{1}{2} \operatorname{Tr}\left[D^{2} \varphi(x)\right]+\langle x, A D \varphi(x)\rangle, \quad \varphi \in \mathcal{E}_{A}(H), x \in H .
$$

Notice that since $D \varphi(x) \in D(A)$, we have $L_{0} \varphi \in \mathcal{C}_{b, 1}(H)$. The next result follows by Proposition 6.2 of [21|21,21]

Proposition 5.2. For any $\varphi \in \mathcal{E}_{A}(H)$ we have $\varphi \in D\left(L, \mathcal{C}_{b, 1}(H)\right)$ and

$$
L \varphi(x)=L_{0} \varphi(x), \quad x \in H .
$$

The set $\mathcal{E}_{A}(H)$ is a $\pi$-core for $\left(L, D\left(L, \mathcal{C}_{b, 1}(H)\right)\right)$, and for any $\varphi \in D\left(L, \mathcal{C}_{b, 1}(H)\right)$ there exist $m \in \mathbb{N}$ and an $m$-indexed sequence $\left(\varphi_{n_{1}, \ldots, n_{m}}\right)_{n_{1}, \ldots, n_{m} \in \mathbb{N}} \subset \mathcal{E}_{A}(H)$ such that

$$
\begin{aligned}
& \lim _{n_{1} \rightarrow \infty} \cdots \lim _{n_{m} \rightarrow \infty} \frac{\varphi_{n_{1}, \ldots, n_{m}}}{1+|\cdot|_{2}} \stackrel{\pi}{=} \frac{\varphi}{1+|\cdot|_{2}}, \\
& \lim _{n_{1} \rightarrow \infty} \cdots \lim _{n_{m} \rightarrow \infty} \frac{L_{0} \varphi_{n_{1}, \ldots, n_{m}}}{1+|\cdot|_{2}} \stackrel{\frac{\pi}{=}}{=\frac{L \varphi}{1+|\cdot|_{2}}} .
\end{aligned}
$$


Finally, if $\varphi \in D\left(L, \mathcal{C}_{b, 1}(H)\right) \cap C_{b}^{1}(H)$ we can choose the sequence in such a way that (21), (22) hold and

$$
\lim _{n_{1} \rightarrow \infty} \cdots \lim _{n_{m} \rightarrow \infty}\left\langle D \varphi_{n_{1}, \ldots, n_{m}}, h\right\rangle \stackrel{\pi}{=}\langle D \varphi, h\rangle,
$$

for any $h \in H$.

Proof. Proposition 6.2 of [21|21,21] shows that (20) holds when the OU semigroup and the corresponding generator is considered in the space con uniformly continuous functions. However, one can see that the all the approximations hold also in $\mathcal{C}_{b, 1}(H)$.

The following results are proved in [21|21,21] in spaces of uniformly continuous functions instead of $\mathcal{C}_{b, 1}(H)$. However, one can see that the proof can be easy adapted to $\mathcal{C}_{b, 1}(H)$.

Proposition 5.3. Let $R_{t}, t \geq 0$ be the OU semigroup (16) in the space $\mathcal{C}_{b, 1}(H)$ and let $\left(L, D\left(L, \mathcal{C}_{b, 1}(H)\right)\right)$ be its infinitesimal generators, defined by (18). Then

(i) $R_{t} \in \mathcal{L}\left(C_{b, 1}(H)\right)$ and $R_{t} R_{s}=R_{t+s}$, for any $t, s \geq 0$;

(ii) for any $\varphi \in C_{b, 1}(H)$ and any sequence $\left(\varphi_{n}\right)_{n \in \mathbb{N}} \subset C_{b, 1}(H)$ such that

$$
\lim _{n \rightarrow \infty} \frac{\varphi_{n}}{1+|\cdot|} \stackrel{\pi}{=} \frac{\varphi}{1+|\cdot|}
$$

we have, for any $t \geq 0$,

$$
\lim _{n \rightarrow \infty} \frac{P_{t} \varphi_{n}}{1+|\cdot|} \stackrel{\pi}{=} \frac{P_{t} \varphi}{1+|\cdot|}
$$

(iii) for any $\varphi \in D\left(L, \mathcal{C}_{b, 1}(H)\right)$, we have $\left.R_{t} \varphi \in D\left(L, \mathcal{C}_{b, 1}(H)\right)\right)$ and $L R_{t} \varphi=$ $R_{t} L \varphi, t \geq 0$

(iv) for any $\varphi \in D\left(L, \mathcal{C}_{b, 1}(H)\right), x \in H$, the map $[0, \infty) \rightarrow \mathbb{R}, t \mapsto R_{t} \varphi(x)$ is continuously differentiable and $(d / d t) R_{t} \varphi(x)=R_{t} L \varphi(x)$;

(v) for any $\lambda>\omega_{0}$ the linear operator $R(\lambda, L)$ on $\mathcal{C}_{b, 1}(H)$ defined by

$$
R(\lambda, L) f(x)=\int_{0}^{\infty} e^{-\lambda t} R_{t} f(x) d t, \quad f \in \mathcal{C}_{b, 1}(H), x \in H
$$

satisfies, for any $f \in C_{b, 1}(H)$

$$
\begin{gathered}
R(\lambda, L) \in \mathcal{L}\left(\mathcal{C}_{b, 1}(H)\right), \\
R(\lambda, L) f \in D\left(L, \mathcal{C}_{b, 1}(H)\right), \quad(\lambda I-L) R(\lambda, L) f=f .
\end{gathered}
$$

We call $R(\lambda, L)$ the resolvent of $L$ at $\lambda$; 
(vi) for any $\varphi \in \mathcal{C}_{b, 1}(H), t>0$, the function

$$
H \rightarrow \mathbb{R}, \quad x \mapsto \int_{0}^{t} R_{s} \varphi(x) d s
$$

belongs to $D\left(L, \mathcal{C}_{b, 1}(H)\right)$, and it holds

$$
L\left(\int_{0}^{t} R_{s} \varphi d s\right)=R_{t} \varphi-\varphi .
$$

We now study the OU semigroup $R_{t}$ in the space $C_{b, V}\left(L^{6}(0,1)\right)$. Proposition 5.1 implies that for any $T>0$ there exists $c_{T}>0$ such that

$$
\mathbb{E}\left[\sup _{t \in[0, T]} V(Z(s, x))\right] \leq c_{T}(1+V(x)) .
$$

Clearly, (24) shows that $R_{t}$ acts on $\mathcal{C}_{b, V}\left(L^{6}(0,1)\right)$. It is obvious that all the results of Proposition 4.2 holds also for the OU semigroup $R_{t}, t \geq 0$. We define the infinitesimal generator of $R_{t}, t \geq 0$ in $\mathcal{C}_{b, V}\left(L^{6}(0,1)\right)$ by setting

$$
\left\{\begin{array}{l}
D\left(L_{V}, \mathcal{C}_{b, V}\left(L^{6}(0,1)\right)\right)=\left\{\varphi \in \mathcal{C}_{b, V}\left(L^{6}(0,1)\right): \exists g \in \mathcal{C}_{b, V}\left(L^{6}(0,1)\right),\right. \\
\left.\lim _{t \rightarrow 0^{+}} \frac{R_{t} \varphi(x)-\varphi(x)}{t}=g(x), \forall x \in L^{6}(0,1), \sup _{t \in(0,1)}\left\|\frac{R_{t} \varphi-\varphi}{t}\right\|_{0, V}<\infty\right\} \\
L_{V} \varphi(x)=\lim _{t \rightarrow 0^{+}} \frac{R_{t} \varphi(x)-\varphi(x)}{t}, \quad \varphi \in D\left(L_{V}, \mathcal{C}_{b, V}\left(L^{6}(0,1)\right)\right), x \in L^{6}(0,1) .
\end{array}\right.
$$

Remark 5.4. Since all the results of Proposition 4.2 hold for the OU semigroup, it follows that all the results of Proposition 4.3 hold for the OU semigroup and its infinitesimal generator in $\mathcal{C}_{b, V}\left(L^{6}(0,1)\right)$.

Proposition 5.5. We have $\mathcal{E}_{A}(H) \subset D\left(L_{V}, \mathcal{C}_{b, V}\left(L^{6}(0,1)\right)\right)$, and $L_{V} \varphi(x)=$ $L \varphi(x)=L_{0} \varphi$, for any $\varphi \in \mathcal{E}_{A}(H), x \in L^{6}(0,1)$.

Proof. Since $\mathcal{C}_{b, 1}(H) \subset \mathcal{C}_{b, V}\left(L^{6}(0,1)\right)$ with continuous embedding, it follows $D\left(L, \mathcal{C}_{b, 1}(H)\right) \subset D\left(L_{V}, \mathcal{C}_{b, V}\left(L^{6}(0,1)\right)\right)$. Then by Proposition 5.5 we have $\mathcal{E}_{A}(H) \subset \mathcal{C}_{b, V}\left(L^{6}(0,1)\right)$ and $L_{V} \varphi(x)=L_{0} \varphi(x), \varphi \in \mathcal{E}_{A}(H), x \in L^{6}(0,1)$.

We conclude this section by the following formula, which will be often recalled throughout the paper

$$
\left\langle D R_{t} \varphi(x), h\right\rangle=R_{t}\left(\left\langle D \varphi, e^{t A} h\right\rangle\right)(x), \quad t \geq 0, \varphi \in \mathcal{C}_{b}^{1}(H), x, h \in H .
$$

Clearly, by the above formula it follows that $\mathcal{C}_{b}^{1}(H)$ is stable under $R_{t}$. 


\section{The approximated problem}

It is convenient to consider the usual Galerkin approximations of equation (2). For any $m \in \mathbb{N}$ we define

$$
b_{m}(x)=P_{m} b\left(P_{m} x\right), \quad x \in H
$$

where

$$
P_{m}=\sum_{i=1}^{m} e_{i} \otimes e_{i}, \quad m \in \mathbb{N} .
$$

We consider the approximating problem

$$
\left\{\begin{array}{l}
d X^{m}(t)=\left(A X^{m}(t)+b_{m}\left(X^{m}(t)\right) d t+d W(t)\right. \\
X^{m}(0)=x
\end{array}\right.
$$

By setting $Y^{m}(t, x)=X^{m}(t, x)-W_{A}(t)$, the corresponding mild form is

$$
Y^{m}(t, x)=e^{t A} x+\frac{1}{2} \int_{0}^{t} e^{(t-s) A} P_{m} D_{\xi}\left(P_{m}\left(Y^{m}(s, x)+W_{A}(s)\right)\right)^{2} d s,
$$

Since for any $m \in \mathbb{N}$ the identity

$$
\left\langle b_{m}(x), x\right\rangle=0, \quad x \in H
$$

holds, all the estimates of Proposition 1.1, 6.2 are uniform on $m$ and we have the following result.

Theorem 6.1. For any $x \in L^{p}(0,1), p \in[2, \infty)$ there exists a unique mild solution $X^{m} \in L^{p}(0,1)$ of equation (27). Moreover, for any $x_{0} \in L^{p}(0,1)$, $\delta>0$ and $T>0$

$$
\lim _{m \rightarrow \infty} \sup _{\substack{x-x_{0} \mid p<\delta \\ t \in[0, T]}}\left|X^{m}(t, x)-X(t, x)\right|_{p}=0
$$

We denote by $P_{t}^{m}$ the transition semigroup

$$
P_{t}^{m} \varphi(x)=\mathbb{E}\left[\varphi\left(X^{m}(t, x)\right)\right], \quad t \geq 0, \varphi \in \mathcal{C}_{b, V}\left(L^{6}(0,1)\right), x \in L^{6}(0,1)
$$

By a standard argument, we find that for any $\mathcal{C}_{b, V}\left(L^{6}(0,1)\right)$ we have

$$
\lim _{m \rightarrow \infty} \frac{P_{t}^{m} \varphi}{1+V} \stackrel{\pi}{=} \frac{P_{t} \varphi}{1+V}, \quad t \geq 0
$$


For any $m \in \mathbb{N}$, we define the infinitesimal generator of the semigroup $P_{t}^{m}$, $t \geq 0$ by

$$
\left\{\begin{array}{l}
D\left(K_{m}, \mathcal{C}_{b, V}\left(L^{6}(0,1)\right)\right)=\left\{\varphi \in \mathcal{C}_{b, V}\left(L^{6}(0,1)\right): \exists g \in \mathcal{C}_{b, V}\left(L^{6}(0,1)\right),\right. \\
\left.\lim _{t \rightarrow 0^{+}} \frac{P_{t}^{m} \varphi(x)-\varphi(x)}{t}=g(x), x \in L^{6}(0,1), \sup _{t \in(0,1)}\left\|\frac{P_{t}^{m} \varphi-\varphi}{t}\right\|_{0, V}<\infty\right\} \\
K_{m} \varphi(x)=\lim _{t \rightarrow 0^{+}} \frac{P_{t} \varphi(x)-\varphi(x)}{t}, \quad \varphi \in D\left(K_{m}, \mathcal{C}_{b, V}\left(L^{6}(0,1)\right)\right), x \in L^{6}(0,1) .
\end{array}\right.
$$

It is clear that all the results of Propositions 4.2, 4.3 hold for $P_{t}^{m}, t \geq 0$ and for its infinitesimal generator $\left(K_{m}, D\left(K_{m}, \mathcal{C}_{b, V}\left(L^{6}(0,1)\right)\right)\right)$.

\subsection{The differential $D P_{t}^{m} \varphi$}

Usually, one derives estimates on the differential $D P_{t} \varphi$ directly from the estimates of the differential $X_{x}(t, x)$ of the solution $X(t, x)$. This method cannot be applied here, by the lack of informations about $X_{x}(t, x)$. In $[8], 8$, it is proposed to consider a Kolmogorov operator with an additional potential term

$$
K_{0}^{\prime} \varphi(x)=K_{0} \varphi(x)-c|x|_{4}^{4} \varphi(x), \quad \varphi \in \mathcal{E}_{A}(H)
$$

and the corresponding semigroup given by the Feynman-Kac formula

$$
S_{t} \varphi(x)=\mathbb{E}\left[e^{-c \int_{0}^{t}|X(s, x)|_{4}^{4} d s} \varphi(X(t, x))\right] .
$$

By using a generalization of the Bismut-Elworthy formula (see [12]12,12]) and some estimates on $X_{x}(t, x)$ the authors are able to get estimates on $D S(t) \varphi$. Then, by the formula

$$
P_{t} \varphi=S_{t} \varphi+c \int_{0}^{t} S_{t-s}\left(|\cdot|{ }_{4}^{4} \varphi\right) d s
$$

they get estimates on $D P_{t} \varphi$.

This method has been successfully implemented to get solutions for the 3D-Navier-Stokes equation (see [717, 7], 15115, 15). It has been also used to get smoothing properties of the differential $D P_{t} \varphi$, with applications to control problems (see, for instance, [55, 5], [66, 6], [22[22, 22])

The following result is proved in Proposition 3.6 of $[8], 8]$.

Proposition 6.2. There exists $\omega_{1}>0$ such that for any $m \in \mathbb{N}, t>0$ and $\varphi \in \mathcal{C}_{b}^{1}(H)$ with $D \varphi \in C_{b}\left(H ; H^{1}(0,1)\right)$ we have $D P_{t}^{m} \varphi(x) \in H^{1}(0,1)$ and

$$
\left|D P_{t}^{m} \varphi(x)\right|_{H^{1}(0,1)} \leq\left(\|D \varphi\|_{C_{b}\left(H ; H^{1}(0,1)\right)}+c\|\varphi\|_{0}\right)\left(1+|x|_{6}\right)^{8} e^{\omega_{1} t}
$$


The following two results are essential for the proof of Theorem 7.1 .

Proposition 6.3. Take $\lambda>\omega_{0}, \omega_{1}$, where $\omega_{0}$ is as in Proposition 4.2 and $\omega_{1}$ is as in Proposition 6.2. Let $f \in \mathcal{E}_{A}(H)$ and, for $m \in \mathbb{N}$ consider the function

$$
L^{6}(0,1) \rightarrow \mathbb{R}, \quad x \mapsto \varphi(x)=\int_{0}^{\infty} e^{-\lambda t} P_{t}^{m} f(x) d t
$$

Then

(i) $\varphi$ is continuous, bounded and Fréchet differentiable in any $x \in L^{6}(0,1)$ with continuous differential $D \varphi \in \mathcal{C}\left(L^{6}(0,1) ; H^{1}(0,1)\right)$. Moreover, it holds

$$
|D \varphi(x)|_{H^{1}(0,1)} \leq \frac{1}{\lambda-\omega_{1}}\left(\|D f\|_{C_{b}\left(H ; H^{1}(0,1)\right)}+c\|f\|_{0}\right)\left(1+|x|_{6}\right)^{8}
$$

(ii) $\varphi$ belongs to $D\left(L_{V}, \mathcal{C}_{b, V}\left(L^{6}(0,1)\right)\right) \cap D\left(K_{m}, \mathcal{C}_{b, V}\left(L^{6}(0,1)\right)\right)$ and

$$
K_{m} \varphi(x)=L_{V} \varphi(x)-\frac{1}{2}\left\langle D_{\xi} P_{m} D \varphi(x),\left(P_{m} x\right)^{2}\right\rangle, \quad \forall x \in L^{6}(0,1) .
$$

Proof. Notice that the mild solution of (27) is defined for any $x \in L^{2}(0,1)=$ $H$ (cfr. [3], 3]). Then, the transition semigroup $P_{t}$ can be defined in $\mathcal{C}_{b}(H)$. So, since $f \in C_{b}(H)$, it follows $\varphi \in \mathcal{C}_{b}(H)$. By Proposition (6.2) we have

$$
\begin{aligned}
|D \varphi(x)|_{H^{1}(0,1)} & \leq \int_{0}^{\infty} e^{-\lambda t}\left|D P_{t} f(x)\right|_{H^{1}(0,1)} d t \\
& \leq \int_{0}^{\infty} e^{-\left(\lambda-\omega_{1}\right) t} d t\left(\|D f\|_{C_{b}\left(H ; H^{1}(0,1)\right)}+c\|f\|_{0}\right)\left(1+|x|_{6}\right)^{8}
\end{aligned}
$$

and (31) follows. Still by (31) we get $D \varphi \in \mathcal{C}\left(L^{6}(0,1) ; H^{1}(0,1)\right)$. Indeed, for any $x, h \in L^{6}(0,1)$,

$$
\begin{gathered}
\left|D \varphi(x+h)-D_{\xi} D \varphi(x)\right|_{H^{1}(0,1)} \\
\leq \frac{1}{\lambda-\omega_{1}}\left(\|D f(\cdot+h)-D f(\cdot)\|_{C_{b}\left(H ; H^{1}(0,1)\right)}+c\|f(\cdot+h)-f(\cdot)\|_{0}\right)\left(1+|x|_{6}\right)^{8}
\end{gathered}
$$

Since $f \in C_{b}(H)$, and $D f \in C_{b}\left(H ; H^{1}(0,1)\right)$, by uniform continuity it follows $|D \varphi(x+h)-D \varphi(x)|_{H^{1}(0,1)} \rightarrow 0$ as $|h|_{6} \rightarrow 0$. This concludes the proof of (i).

Let us prove (ii). Since the semigroup $P_{t}^{m}, t \geq 0$ satisfies the statements of Proposition 4.2, it follows that its infinitesimal generator $K_{m}$ enjoys the statements of Proposition 4.3. In particular, we have $\varphi=R\left(\lambda, K_{m}\right) f$ and therefore $\varphi \in D\left(K_{m}, \mathcal{C}_{b, V}\left(L^{6}(0,1)\right)\right)$. Then we have to show that $\varphi \in$ 
$D\left(L_{V}, \mathcal{C}_{b, V}\left(L^{6}(0,1)\right)\right)$. Now let $R_{t}, t \geq 0$ be the OU semigroup (16) and let $\left(L_{V}, D\left(L_{V}, \mathcal{C}_{b, V}\left(L^{6}(0,1)\right)\right)\right)$ be its infinitesimal generator in $\mathcal{C}_{b, V}\left(L^{6}(0,1)\right)$. Fix $x \in L^{6}(0,1), T>0$ and for $t \in[0, T]$ set $X^{m}(t)=X^{m}(t, x), Z(t)=$ $Z(t, x)$. By (28), (14) we have

$$
X^{m}(t)=Z(t)+\frac{1}{2} \int_{0}^{t} e^{(t-s) A} P_{m} D_{\xi}\left(P_{m} X^{m}(s)\right)^{2} d s
$$

and consequently

$$
P_{t}^{m} \varphi(x)=\mathbb{E}\left[\varphi\left(X^{m}(t)\right)\right]=\mathbb{E}\left[\varphi\left(Z(t)+\frac{1}{2} \int_{0}^{t} e^{(t-s) A} P_{m} D_{\xi}\left(P_{m} X^{m}(s)\right)^{2} d s\right)\right] .
$$

Notice that since $f \in C_{b}^{1}(H)$, by (31) we get that the function $L^{6}(0,1) \rightarrow \mathbb{R}$, $x \mapsto D \varphi(x)$ is continuous. Then, by Taylor formula we have

$$
\begin{gathered}
R_{t} \varphi(x)-\varphi(x)=P_{t}^{m} \varphi(x)-\varphi(x) \\
+\frac{1}{2} \mathbb{E}\left[\int_{0}^{1}\left\langle D \varphi\left(\xi X^{m}(t)+(1-\xi) Z(t)\right), \int_{0}^{t} e^{(t-s) A} P_{m} D_{\xi}\left(P_{m} X^{m}(s)\right)^{2} d s\right\rangle d \xi\right]
\end{gathered}
$$

We claim that

$$
\begin{aligned}
\lim _{t \rightarrow 0^{+}} \frac{1}{t} \mathbb{E}\left[\int _ { 0 } ^ { 1 } \left\langleD \varphi \left(\xi X^{m}(t)\right.\right.\right. & \left.\left.+(1-\xi) Z(t)), \int_{0}^{t} e^{(t-s) A} P_{m} D_{\xi}\left(P_{m} X^{m}(s)\right)^{2} d s\right\rangle d \xi\right] \\
= & -\left\langle D_{\xi} P_{m} D \varphi(x),\left(P_{m} x\right)^{2}\right\rangle
\end{aligned}
$$

holds. By Theorem 3.1, for any $T>0$ we can write

$$
\begin{aligned}
X(t) & =x+\theta_{1}(t) \\
Z(t) & =x+\theta_{2}(t), \quad t \in[0, T]
\end{aligned}
$$

where $\theta_{1}(t), \theta_{2}(t): \Omega \rightarrow H, t \in[0, T]$ are random variables such that $\theta_{1}, \theta_{2} \in$ $C([0, T] ; H) \mathbb{P}$-a.s. and $\theta_{1}(0)=\theta_{2}(0)=0$. On the other hand, by Proposition 6.3 we can write

$$
D \varphi(x+z)=D \varphi(x)+\eta(z), \quad z \in H
$$

where $\eta \in \mathcal{C}\left(H, H^{1}(0,1)\right)$ and $\eta(0)=0$. With these notations we have

$$
\begin{aligned}
D \varphi\left(\xi X^{m}(t)+(1-\xi) Z(t)\right) & =D \varphi\left(x+\xi \theta_{1}(t)+(1-\xi) \theta_{2}(t)\right) \\
& =D \varphi(x)+\eta\left(\xi \theta_{1}(t)+(1-\xi) \theta_{2}(t)\right) .
\end{aligned}
$$


Then

$$
\begin{gathered}
\lim _{t \rightarrow 0^{+}} \sup _{\xi \in[0,1]}\left|D \varphi\left(\xi X^{m}(t)+(1-\xi) Z(t)\right)-D \varphi(x)\right|_{H^{1}(0,1)}= \\
\quad=\lim _{t \rightarrow 0^{+}} \sup _{\xi \in[0,1]}\left|\eta\left(\xi \theta_{1}(t)+(1-\xi) \theta_{2}(t)\right)\right|_{H^{1}(0,1)}=0 .
\end{gathered}
$$

For any $t>0$ we have

$$
\begin{aligned}
&\left|\frac{1}{t} \int_{0}^{t} e^{(t-s) A} P_{m} D_{\xi}\left(P_{m} X^{m}(s)\right)^{2} d s-P_{m} D_{\xi}\left(\left(P_{m} x\right)^{2}\right)\right|_{W^{-1,2}(0,1)} \\
& \leq \frac{1}{t} \int_{0}^{t}\left|e^{(t-s) A} P_{m} D_{\xi}\left(\left(P_{m} X^{m}(s)\right)^{2}-\left(P_{m} x\right)^{2}\right)\right|_{W^{-1,2}(0,1)} d s \\
& \quad+\frac{1}{t} \int_{0}^{t}\left|e^{(t-s) A} P_{m} D_{\xi}\left(P_{m} x\right)^{2}-P_{m} D_{\xi}\left(P_{m} x\right)^{2}\right|_{W^{-1,2}(0,1)} d s .
\end{aligned}
$$

The first term on the right-hand side is bounded by

$$
\begin{aligned}
& \frac{1}{t} \int_{0}^{t}\left|P_{m} D_{\xi}\left(\left(P_{m} X^{m}(s)\right)^{2}-\left(P_{m} x\right)^{2}\right)\right|_{W^{-1,2}(0,1)} d s \\
& \quad \leq \frac{1}{t} \int_{0}^{t}\left|\left(P_{m} X^{m}(s)\right)^{2}-\left(P_{m} x\right)^{2}\right|_{2} d s \\
& \quad \leq \frac{1}{t} \int_{0}^{t}\left|X^{m}(s)-x\right|_{2}\left|X^{m}(s)+x\right|_{2} d s
\end{aligned}
$$

Since $X^{m} \in C([0, T] ; H) \mathbb{P}$-a.s., it follows

$$
\lim _{t \rightarrow 0^{+}} \frac{1}{t} \int_{0}^{t}\left|e^{(t-s) A} P_{m} D_{\xi}\left(\left(P_{m} X^{m}(s)\right)^{2}-\left(P_{m} x\right)^{2}\right)\right|_{W^{-1,2}(0,1)} d s=0, \quad \mathbb{P} \text {-a.s. }
$$

Since the semigroup $e^{t A}, t \geq 0$ can be est ended to a strongly continuous semigroup in $W^{-1,2}(0,1)$, for the last term of (36) it holds

$$
\lim _{t \rightarrow 0^{+}} \frac{1}{t} \int_{0}^{t}\left|e^{(t-s) A} P_{m} D_{\xi}\left(P_{m} x\right)^{2}-P_{m} D_{\xi}\left(P_{m} x\right)^{2}\right|_{W^{-1,2}(0,1)} d s=0 .
$$

Hence, by (36) we have

$$
\lim _{t \rightarrow 0^{+}}\left|\frac{1}{t} \int_{0}^{t} e^{(t-s) A} P_{m} D_{\xi}\left(P_{m} X^{m}(s)\right)^{2} d s-P_{m} D_{\xi}\left(P_{m} x\right)^{2}\right|_{W^{-1,2}(0,1)}=0, \quad \mathbb{P} \text {-a.s. }
$$

This, together with (32) and an integration by parts, implies

$$
\lim _{t \rightarrow 0^{+}} \frac{1}{t} \int_{0}^{1}\left\langle D \varphi\left(\xi X^{m}(t)+(1-\xi) Z(t)\right), \int_{0}^{t} e^{(t-s) A} P_{m} D_{\xi}\left(P_{m} X^{m}(s)\right)^{2} d s\right\rangle d \xi
$$




$$
=\left\langle D \varphi(x), P_{m} D_{\xi}\left(P_{m} x\right)^{2}\right\rangle=-\left\langle D_{\xi} P_{m} D \varphi(x),\left(P_{m} x\right)^{2}\right\rangle, \quad \mathbb{P} \text {-a.s. }
$$

In order to obtain (35), it is sufficient to show that the terms in the above limit are dominated by an integrable random variable. Indeed, for any $t \in(0, T]$ we have

$$
\begin{gathered}
\frac{1}{t} \int_{0}^{1}\left\langle D \varphi\left(\xi X^{m}(t)+(1-\xi) Z(t)\right), \int_{0}^{t} e^{(t-s) A} P_{m} D_{\xi}\left(P_{m} X^{m}(s)\right)^{2} d s\right\rangle d \xi \\
\leq \frac{1}{t}\left|\int_{0}^{1} D \varphi\left(\xi X^{m}(t)+(1-\xi) Z(t)\right) d \xi\right|_{H^{1}(0,1)} \\
\quad \times\left|\int_{0}^{t} e^{(t-s) A} P_{m} D_{\xi}\left(P_{m} X^{m}(s)\right)^{2} d s\right|_{W^{-1,2}(0,1)} \\
\leq \int_{0}^{1}\left|D \varphi\left(\xi X^{m}(t)+(1-\xi) Z(t)\right)\right|_{H^{1}(0,1)} d \xi \\
\times \frac{1}{t} \int_{0}^{t}\left|e^{(t-s) A} P_{m} D_{\xi}\left(P_{m} X^{m}(s)\right)^{2}\right|_{W^{-1,2}(0,1)} d s \\
\leq I_{1}(t) \times I_{2}(t) .
\end{gathered}
$$

Set

$$
C_{\varphi}=\left(\frac{\|D f\|_{C_{b}\left(H ; H^{1}(0,1)\right)}+c\|f\|_{0}}{\lambda-\omega_{1}}\right) .
$$

By (31) we have

$$
\begin{aligned}
\int_{0}^{1} & \left|D \varphi\left(\xi X^{m}(t, x)+(1-\xi) Z(t, x)\right)\right|_{H^{1}(0,1)} d \xi \\
& \leq C_{\varphi}\left(1+\left|\xi X^{m}(t, x)+(1-\xi) Z(t, x)\right|_{6}^{8}\right) \\
& \leq C_{\varphi} \int_{0}^{1}\left(1+\xi\left|X^{m}(t, x)\right|_{6}^{8}+(1-\xi)|Z(t, x)|_{6}^{8}\right) d \xi \\
& \leq C_{\varphi}\left(1+\sup _{t \in[0, T]}\left|X^{m}(t, x)\right|_{6}^{8}+\sup _{t \in[0, T]}|Z(t, x)|_{6}^{8}\right)
\end{aligned}
$$

Here we have used the convexity of the function $z \rightarrow|z|_{6}^{8}$. For $I_{2}(t)$ we have

$$
\begin{aligned}
I_{2}(t) & \leq \frac{c}{t} \int_{0}^{t}\left|\left(P_{m} X^{m}(s)\right)^{2}\right|_{2} d s \\
& \leq \frac{c}{t} \int_{0}^{t}\left|X^{m}(s)\right|_{4}^{2} d s \leq c \sup _{t \in[0, T]}\left|X^{m}(t)\right|_{4}^{2}
\end{aligned}
$$

Then, for any $t \in(0, T]$ we have 


$$
\begin{aligned}
& \frac{1}{t}\left|\int_{0}^{1}\left\langle D \varphi\left(\xi X^{m}(t)+(1-\xi) Z(t)\right), \int_{0}^{t} e^{(t-s) A} P_{m} D_{\xi}\left(P_{m} X^{m}(s)\right)^{2} d s\right\rangle d \xi\right| \\
& \leq c C_{\varphi}\left(1+\sup _{t \in[0, T]}\left|X^{m}(t, x)\right|_{6}^{8}+\sup _{t \in[0, T]}|Z(t, x)|_{6}^{8}\right)\left(\sup _{t \in[0, T]}\left|X^{m}(t)\right|_{4}^{2}\right)
\end{aligned}
$$

Notice that by Propositions 1.1, (5.1) the random variable

$$
g(x):=c C_{\varphi}\left(1+\sup _{t \in[0, T]}\left|X^{m}(t, x)\right|_{6}^{8}+\sup _{t \in[0, T]}|Z(t, x)|_{6}^{8}\right)\left(\sup _{t \in[0, T]}\left|X^{m}(t)\right|_{4}^{2}\right)
$$

belongs to $L^{1}(\Omega, \mathbb{P})$ and

$$
\mathbb{E}[g(x)] \leq C\left(1+|x|_{6}^{8}|x|_{4}^{2}\right)
$$

for some $C>0$. Consequently, since for any $t \in(0, T]$

$\frac{1}{t}\left|\int_{0}^{1}\left\langle D \varphi\left(\xi X^{m}(t)+(1-\xi) Z(t)\right), \int_{0}^{t} e^{(t-s) A} P_{m} D_{\xi}\left(P_{m} X^{m}(s)\right)^{2} d s\right\rangle d \xi\right| \leq g(x)$,

by the dominated convergence theorem and by (37) follows (34) as claimed.

By (33), (34) and by the fact that $\varphi \in D\left(K_{m}, \mathcal{C}_{b, V}\left(L^{6}(0,1)\right)\right)$ we obtain

$$
\lim _{t \rightarrow 0^{+}} \frac{R_{t} \varphi(x)-\varphi(x)}{t}=K_{m} \varphi+\frac{1}{2}\left\langle D_{\xi} P_{m} D \varphi(x),\left(P_{m} x\right)^{2}\right\rangle, \quad \forall x \in L^{6}(0,1) .
$$

Now, by (38), (39), (40) we have

$$
\begin{aligned}
\sup _{t \in(0, T]}\left|\frac{R_{t} \varphi(x)-\varphi(x)}{t}\right| & \leq \sup _{t \in(0, T]}\left|\frac{P_{t}^{m} \varphi(x)-\varphi(x)}{t}\right|+\mathbb{E}[g(x)] \\
& \leq c(1+V(x))
\end{aligned}
$$

since $\varphi \in D\left(K_{m}, \mathcal{C}_{b, V}\left(L^{6}(0,1)\right)\right.$. This implies $\varphi \in D\left(L_{V}, \mathcal{C}_{b, V}\left(L^{6}(0,1)\right)\right)$ and (32) follows.

Proposition 6.4. Fix $m \in \mathbb{N}, f \in \mathcal{E}_{A}(H)$ and let $\varphi$ be as in Proposition 6.3. Then, there exist $k \in \mathbb{N}$ and a $k$-indexed sequence $\left(\varphi_{n_{1}, \ldots, n_{k}}\right)_{n_{1} \in \mathbb{N}, \ldots, n_{k} \in \mathbb{N}} \subset$ $\mathcal{E}_{A}(H)$ such that

$$
\begin{gathered}
\lim _{n_{1} \rightarrow \infty} \cdots \lim _{n_{k} \rightarrow \infty} \frac{\varphi_{n_{1}, \ldots, n_{k}}}{1+V} \stackrel{\pi}{=} \frac{\varphi}{1+V} \\
\lim _{n_{1} \rightarrow \infty} \cdots \lim _{n_{k} \rightarrow \infty} \frac{L_{0} \varphi_{n_{1}, \ldots, n_{k}}}{1+V} \stackrel{\pi}{=} \frac{L \varphi}{1+V}
\end{gathered}
$$

and, for any $h \in H$

$$
\lim _{n_{1} \rightarrow \infty} \cdots \lim _{n_{k} \rightarrow \infty} \frac{\left\langle D_{\xi} D \varphi_{n_{1}, \ldots, n_{k}}, h\right\rangle}{\left(1+\left.|\cdot|\right|_{6} ^{8}\right)} \stackrel{\pi}{=} \frac{\left\langle D_{\xi} D \varphi, h\right\rangle}{\left(1+\left.|\cdot|\right|_{6} ^{8}\right)}
$$


Proof. Set

$$
\psi_{p}(x)=\left(1+p^{-1}\left|e^{\frac{1}{p} A} x\right|_{6}^{8}\right)^{-1} \varphi\left(e^{\frac{1}{p} A} x\right), \quad x \in H, p \in \mathbb{N} .
$$

Clearly,

$$
\lim _{p \rightarrow \infty} \frac{\psi_{p}}{1+|\cdot|_{6}^{8}} \stackrel{\pi}{=} \frac{\varphi}{1+|\cdot|_{6}^{8}}
$$

By the well known properties of the heat semigroup, $e^{\frac{1}{p} A} x \in L^{6}(0,1)$, for any $x \in H$. Then, since by Proposition 6.3 we have $\varphi \in \mathcal{C}_{b}\left(L^{6}(0,1)\right)$, it follows that $\psi_{p}: H \rightarrow \mathbb{R}$ is bounded. Moreover, an easy computation show that $\psi_{p}$ is continuous. Then, $\psi_{p} \in \mathcal{C}_{b}(H)$. A standard computation show

$$
\left\langle D \psi_{p}(x), h\right\rangle=\frac{\left\langle D \varphi\left(e^{\frac{1}{p} A} x\right), e^{\frac{1}{p} A} h\right\rangle}{1+p^{-1}\left|e^{\frac{1}{p} A} x\right|_{6}^{8}}-\frac{8 \varphi\left(e^{\frac{1}{p} A} x\right)\left|e^{\frac{1}{p} A} x\right|_{6}^{7}\left\langle\left(e^{\frac{1}{p} A} x\right)^{5}, e^{\frac{1}{p} A} h\right\rangle}{p\left(1+p^{-1}\left|e^{\frac{1}{p} A} x\right|_{6}^{8}\right)^{2}},
$$

$x, h \in H$. Hence, by taking into account (31), there exists $c_{f}>0$, depending on $f$, such that for any $x \in L^{6}(0,1)$ we have

$$
\begin{aligned}
\left|\left\langle D \psi_{p}(x), h\right\rangle\right| & \leq \frac{\left|D \varphi\left(e^{\frac{1}{p} A} x\right)\right|_{2}\left|e^{\frac{1}{p} A} h\right|_{2}}{1+p^{-1}\left|e^{\frac{1}{p} A} x\right|_{6}^{8}}+\frac{8\|\varphi\|_{0}\left|e^{\frac{1}{p} A} x\right|_{6}^{7}\left|e^{\frac{1}{p} A} x\right|_{6}^{3}\left|e^{\frac{1}{p} A} h\right|_{L^{6 / 5}}}{p\left(1+p^{-1}\left|e^{\frac{1}{p} A} x\right|_{6}^{8}\right)^{2}} \\
& \leq\left(\frac{c_{f}\left(1+\left|e^{\frac{1}{p} A} x\right|_{6}^{8}\right)}{\left(1+p^{-1}\left|e^{\frac{1}{p} A} x\right|_{6}^{8}\right)\left(\lambda-\omega_{1}\right)}+\frac{2\|\|_{0}\left|e^{\frac{1}{p} A} x\right|_{6}^{10}}{p\left(1+p^{-1}\left|e^{\frac{1}{p} A} x\right|_{6}^{8}\right)^{2}}\right)\left|e^{\frac{1}{p} A} h\right|_{2} \\
& \leq\left(p \frac{c_{f}}{\lambda-\omega_{1}}+2\|\varphi\|_{0}\right)|h|_{2} .
\end{aligned}
$$

Then, $\psi_{p}$ is Fréchet differentiable in any $x \in H$ and its differential is bounded. An easy but tedious computation shows that $D \psi_{p}: H \rightarrow \mathcal{L}(H)$ is continuous. Therefore, $\psi_{p} \in \mathcal{C}_{b}^{1}(H)$. In addition, as easily checked, by formula (45) and by (31) (which gives the bound for $D \varphi$ ) it follows

$$
\lim _{p \rightarrow \infty} \frac{\left\langle D \psi_{p}, h\right\rangle}{1+|\cdot|_{6}^{8}} \stackrel{\pi}{=} \frac{\langle D \varphi, h\rangle}{1+|\cdot|_{6}^{8}}, \quad \forall h \in H
$$

For any $n_{2}, n_{3} \in \mathbb{N}$, consider the function

$$
\psi_{n_{2}, n_{3}}: H \rightarrow \mathbb{R}, \quad x \mapsto \psi_{n_{2}, n_{3}}(x)=n_{2} \int_{0}^{\frac{1}{n_{2}}} R_{t} \psi_{n_{3}}(x) d t .
$$


By (vi) of Proposition 5.3 we have $\psi_{n_{2}, n_{3}} \in D\left(L, \mathcal{C}_{b, 1}(H)\right)$ and by (26) we have $\psi_{n_{2}, n_{3}} \in C_{b}^{1}(H)$. Then

$$
\psi_{n_{2}, n_{3}} \in D\left(L, \mathcal{C}_{b, 1}(H)\right) \cap \mathcal{C}_{b}^{1}(H), \quad n_{2}, n_{3} \in \mathbb{N} .
$$

Then, by Proposition 5.2 there exists a sequence $\left.11 \psi_{n_{2}, n_{3}, n_{4}}\right\}_{n_{4} \in \mathbb{N}} \subset \mathcal{E}_{A}(H)$ such that

$$
\begin{gathered}
\lim _{n_{4} \rightarrow \infty} \psi_{n_{2}, n_{3}, n_{4}} \stackrel{\pi}{=} \psi_{n_{2}, n_{3}}, \quad \lim _{n_{4} \rightarrow \infty} L_{0} \psi_{n_{2}, n_{3}, n_{4}} \stackrel{\pi}{=} L \psi_{n_{2}, n_{3}} \\
\lim _{n_{4} \rightarrow \infty}\left\langle D \psi_{n_{2}, n_{3}, n_{4}}, h\right\rangle \stackrel{\pi}{=}\left\langle D \psi_{n_{2}, n_{3}}, h\right\rangle, \quad \forall h \in H .
\end{gathered}
$$

Now set

$$
\begin{aligned}
& \varphi_{n_{1}}=R_{\frac{1}{n_{1}}} \varphi \\
& \varphi_{n_{1}, n_{2}}=n_{2} \int_{0}^{\frac{1}{n_{2}}} R_{t+\frac{1}{n_{1}}} \varphi d t, \\
& \varphi_{n_{1}, n_{2}, n_{3}}=n_{2} \int_{0}^{\frac{1}{n_{2}}} R_{t+\frac{1}{n_{1}}} \psi_{n_{3}} d t=R_{\frac{1}{n_{1}}} \psi_{n_{2}, n_{3}}, \\
& \varphi_{n_{1}, n_{2}, n_{3}, n_{4}}=R_{\frac{1}{n_{1}}} \psi_{n_{2}, n_{3}, n_{4}}
\end{aligned}
$$

As easily checked, by the definition of $\varphi_{n_{1}, n_{2}, n_{3}, n_{4}}$ and by (44), (44)

$$
\lim _{n_{1} \rightarrow \infty} \lim _{n_{2} \rightarrow \infty} \lim _{n_{3} \rightarrow \infty} \lim _{n_{4} \rightarrow \infty} \frac{\varphi_{n_{1}, n_{2}, n_{3}, n_{4}}}{1+|\cdot|_{6}^{8}} \stackrel{\pi}{=} \frac{\varphi}{1+|\cdot|_{6}^{8}}
$$

which implies (41).

Let us show (42). Since $\psi_{n_{2}, n_{3}, n_{4}}$, by (19) we have that $\varphi_{n_{1}, n_{2}, n_{3}, n_{4}} \in \mathcal{E}_{A}(H)$ and by Proposition 5.2 we have

$$
L \varphi_{n_{1}, n_{2}, n_{3}, n_{4}}=L_{0} \varphi_{n_{1}, n_{2}, n_{3}, n_{4}}, \quad \forall n_{1}, n_{2}, n_{3}, n_{4} \in \mathbb{N} .
$$

Consequently, by (47) and by (iii) of Proposition 5.3

$$
\begin{aligned}
& \lim _{n_{4} \rightarrow \infty} L_{0} \varphi_{n_{1}, n_{2}, n_{3}, n_{4}}=\lim _{n_{4} \rightarrow \infty} R_{\frac{1}{n_{1}}} L \psi_{n_{2}, n_{3}, n_{4}} \\
& \stackrel{\pi}{=} R_{\frac{1}{n_{1}}} L \psi_{n_{2}, n_{3}}=L R_{\frac{1}{n_{1}}} \psi_{n_{2}, n_{3}}=L \varphi_{n_{1}, n_{2}, n_{3}} .
\end{aligned}
$$

By (vi) Proposition 5.3 we have

$$
L R_{\frac{1}{n_{1}}} \psi_{n_{2}, n_{3}}=n_{2}\left(R_{\frac{1}{n_{1}}+\frac{1}{n_{2}}} \psi_{n_{3}}-R_{\frac{1}{n_{1}}} \psi_{n_{3}}\right) .
$$

\footnotetext{
${ }^{1}$ we assume that the sequence has one index
} 
Therefore

$$
\begin{aligned}
\lim _{n_{2} \rightarrow \infty} \lim _{n_{3} \rightarrow \infty} \frac{L \varphi_{n_{1}, n_{2}, n_{3}}\left(R_{\frac{1}{n_{1}}+\frac{1}{n_{2}}} \psi_{n_{3}}-R_{\frac{1}{n_{1}}} \psi_{n_{3}}\right)}{1+V} \stackrel{\stackrel{\pi}{=} \lim _{n_{2} \rightarrow \infty} \frac{\left(R_{\frac{1}{n_{1}}+\frac{1}{n_{2}}} \varphi-R_{\frac{1}{n_{1}}} \varphi\right)}{1+V}}{ } \\
\stackrel{\frac{\pi}{=}}{\frac{R_{\frac{1}{n_{1}}} L \varphi}{1+V} .}
\end{aligned}
$$

The last equality follows by (v) of Proposition 4.2 and by the fact that $\varphi \in D\left(L_{V}, \mathcal{C}_{b, V}\left(L^{6}(0,1)\right)\right)$. Finally, since

$$
\lim _{n_{1} \rightarrow \infty} \frac{R_{\frac{1}{n_{1}}} L \varphi}{1+V} \stackrel{\pi}{=} \frac{L \varphi}{1+V},
$$

(42) follows.

Let us show (43). Notice that for any $n_{1}, n_{2}, n_{3}, n_{4} \in \mathbb{N}, h \in H_{0}^{1}(0,1)$ we have

$$
\begin{aligned}
\left\langle D \varphi_{n_{1}, n_{2}, n_{3}, n_{4}}(x), D_{\xi} h\right\rangle & =R_{\frac{1}{n_{1}}}\left(\left\langle e^{\frac{1}{n_{1}} A} D \psi_{n_{2}, n_{3}, n_{4}}, D_{\xi} h\right\rangle\right)(x) \\
& =-R_{\frac{1}{n_{1}}}\left(\left\langle D_{\xi} e^{\frac{1}{n_{1}} A} D \psi_{n_{2}, n_{3}, n_{4}}, h\right\rangle\right)(x) .
\end{aligned}
$$

Here we have also used formula (26). By the elementary properties of the heat semigroup, for any $t>0$ the linear operator $D_{\xi} e^{t A}: H_{0}^{1}(0,1) \rightarrow H$, $z \mapsto D_{\xi} e^{t A} z$ is bounded by $\left|D_{\xi} e^{t A} z\right|_{2} \leq c t^{-1 / 2}|z|_{2}$, where $c>0$ is independent of $t$. Then $D_{\xi} e^{\frac{1}{n_{1}} A}: H_{0}^{1}(0,1) \rightarrow H$ can be extended to a linear and bounded operator in $H$, which we still denote by $D_{\xi} e^{\frac{1}{n_{1}} A}$. Then by (48) we have

$$
\lim _{n_{4} \rightarrow \infty}\left\langle D_{\xi} D \varphi_{n_{1}, n_{2}, n_{3}, n_{4}}, h\right\rangle \stackrel{\pi}{=}\left\langle D_{\xi} D \varphi_{n_{1}, n_{2}, n_{3}}, h\right\rangle, \quad \forall h \in H .
$$

By the same argument we find

$$
\lim _{n_{3} \rightarrow \infty}\left\langle D_{\xi} D \varphi_{n_{1}, n_{2}, n_{3}}, h\right\rangle \stackrel{\pi}{=}\left\langle D_{\xi} D \varphi_{n_{1}, n_{2}}, h\right\rangle, \quad \forall h \in H .
$$

Notice now that by definition of $\varphi_{n_{1}, n_{2}}$ we have

$$
\left\langle D_{\xi} D \varphi_{n_{1}, n_{2}}(x), h\right\rangle=R_{\frac{1}{n_{1}}}\left(\left\langle D_{\xi} e^{\frac{1}{n_{1}} A} D \psi_{n_{2}}, h\right\rangle\right)(x), \quad x, h \in H .
$$

Now, since $D_{\xi} e^{\frac{1}{n_{1}} A}: H \rightarrow H$ is linear and bounded, by (46) it follows

$$
\lim _{n_{2} \rightarrow \infty} \frac{\left\langle D_{\xi} e^{\frac{1}{n_{1}} A} D \psi_{n_{2}}, h\right\rangle}{1+|\cdot|_{6}^{8}} \stackrel{\pi}{=} \frac{\left\langle D_{\xi} e^{\frac{1}{n_{1}} A} D \varphi, h\right\rangle}{1+|\cdot|_{6}^{8}} .
$$


Hence, by Proposition 4.2 (cfr. Remark 5.4) we have

$$
\lim _{n_{2} \rightarrow \infty} \frac{\left\langle D_{\xi} D \varphi_{n_{1}, n_{2}}, h\right\rangle}{1+|\cdot|_{6}^{8}} \stackrel{\pi}{=} \frac{\left\langle D_{\xi} D \varphi_{n_{1}}, h\right\rangle}{1+|\cdot|_{6}^{8}}
$$

Finally, by Proposition 4.2 applied to the semigroup $R_{t}, t \geq 0$ we find

$$
\begin{aligned}
\lim _{n_{1} \rightarrow \infty} \frac{\left\langle D_{\xi} D \varphi_{n_{1}}, h\right\rangle}{1+|\cdot|_{6}^{8}} & =\lim _{n_{1} \rightarrow \infty} \frac{R_{\frac{1}{n_{1}}}\left(\left\langle D_{\xi} e^{\frac{1}{n_{1}} A} D \varphi, h\right\rangle\right)}{1+|\cdot|_{6}^{8}} \\
& \stackrel{\frac{\pi}{=}}{\frac{\left\langle D_{\xi} D \varphi, h\right\rangle}{1+|\cdot|_{6}^{8}}}
\end{aligned}
$$

This complete the proof.

\section{Main result: a core for $K$}

In order to prove Theorem 7.1 below we show that $K$ is an extension of $K_{0}$ and that $\left(\lambda I-K_{0}\right)\left(\mathcal{E}_{A}(H)\right)$ is dense, with respect to the $\pi$-convergence, in $\mathcal{C}_{b, V}\left(L^{6}(0,1)\right)$. The proof follows a scheme which has been successfully implemented in [20120, 20] and [20[20, 20]. Here we give all the details. We stress that a crucial role is played by the estimates for the differential $D P_{t}$ given in the previous section.

Theorem 7.1. The operator $\left(K, D\left(K, \mathcal{C}_{b, V}\left(L^{6}(0,1)\right)\right)\right)$ is an extension of $K_{0}$, and for any $\varphi \in \mathcal{E}_{A}(H)$ we have $\varphi \in D\left(K, \mathcal{C}_{b, V}\left(L^{6}(0,1)\right)\right)$ and $K \varphi=K_{0} \varphi$. Finally, the set $\mathcal{E}_{A}(H)$ is a $\pi$-core for $\left(K, D\left(K, \mathcal{C}_{b, V}\left(L^{6}(0,1)\right)\right)\right)$, that is for any $\varphi \in D\left(K, \mathcal{C}_{b, V}\left(L^{6}(0,1)\right)\right)$ there exist $m \in \mathbb{N}$ and an $m$-indexed sequence $\left(\varphi_{n_{1}, \ldots, n_{m}}\right)_{n_{1} \in \mathbb{N}, \ldots, n_{m} \in \mathbb{N}} \subset \mathcal{E}_{A}(H)$ such that

$$
\lim _{n_{1} \rightarrow \infty} \cdots \lim _{n_{m} \rightarrow \infty} \frac{\varphi_{n_{1}, \ldots, n_{m}}}{1+V} \stackrel{\pi}{=} \frac{\varphi}{1+V}
$$

and

$$
\lim _{n_{1} \rightarrow \infty} \cdots \lim _{n_{m} \rightarrow \infty} \frac{K_{0} \varphi_{n_{1}, \ldots, n_{m}}}{1+V} \stackrel{\pi}{=} \frac{K \varphi}{1+V}
$$

We split the proof into two lemmata.

Lemma 7.2. $K$ is an extension of $K_{0}$ and $K \varphi=K_{0} \varphi$ for any $\varphi \in \mathcal{E}_{A}(H)$.

Proof. Take $h \in D(A)$. It is sufficient to show the claim for

$$
\varphi(x)=e^{i\langle x, h\rangle}, \quad x \in L^{6}(0,1) .
$$


Let $\left(L, D\left(L, \mathcal{C}_{b, 1}(H)\right)\right)$ be the infinitesimal generator in $\mathcal{C}_{b, 1}(H)$ of the OrnsteinUhlenbeck semigroup associated to the mild solution of (14) and, for any $m \in \mathbb{N}$, let $\left(K_{m}, D\left(K_{m}, \mathcal{C}_{b, V}\left(L^{6}(0,1)\right)\right)\right)$ be the infinitesimal generator of the semigroup $P_{t}^{m}, t \geq 0$ in $\mathcal{C}_{b, V}\left(L^{6}(0,1)\right)$, as defined in (29), (30). Since $\mathcal{E}_{A}(H) \subset D\left(L, \mathcal{C}_{b}^{1}(H)\right) \cap \mathcal{C}_{b, 1}(H)$, by arguing as for Proposition 6.3 we find that for any $t \geq 0, x \in L^{6}(0,1)$ it holds

$$
\begin{gathered}
P_{t}^{m} \varphi(x)-R_{t} \varphi(x) \\
=\frac{i}{2} \mathbb{E}\left[\int_{0}^{1} \varphi\left(\xi Z(t, x)+(1-\xi) X^{m}(t, x)\right) d \xi\left\langle h, \int_{0}^{t} e^{(t-s) A} P_{m} D_{\xi}\left(P_{m} X^{m}(s, x)\right)^{2} d s\right\rangle\right] \\
=\frac{i}{2} \mathbb{E}\left[\int_{0}^{1} \varphi\left(\xi Z(t, x)+(1-\xi) X^{m}(t, x)\right) d \xi \int_{0}^{t}\left\langle h, e^{(t-s) A} P_{m} D_{\xi}\left(P_{m} X^{m}(s, x)\right)^{2}\right\rangle d s d \xi\right] \\
=-\frac{i}{2} \mathbb{E}\left[\int_{0}^{1} \varphi\left(\xi Z(t, x)+(1-\xi) X^{m}(t, x)\right) d \xi \int_{0}^{t}\left\langle D_{\xi} P_{m} e^{(t-s) A} h,\left(P_{m} X^{m}(s, x)\right)^{2}\right\rangle d s\right] .
\end{gathered}
$$

In the above computation we have used $D \varphi(x)=i \varphi(x) h, x \in L^{6}(0,1)$.

Letting $m \rightarrow \infty$, by Theorem 6.1 we find

$$
\begin{gathered}
P_{t} \varphi(x)-\varphi(x)=R_{t} \varphi(x)-\varphi(x) \\
-\frac{i}{2} \mathbb{E}\left[\int_{0}^{1} \varphi(\xi Z(t, x)+(1-\xi) X(t, x)) d \xi \int_{0}^{t}\left\langle D_{\xi} e^{(t-s) A} h,(X(s, x))^{2}\right\rangle d s\right] .
\end{gathered}
$$

This implies, still by arguing as for Proposition 6.3,

$$
\lim _{t \rightarrow 0^{+}} \frac{P_{t} \varphi(x)-\varphi(x)}{t}=L \varphi(x)-\frac{i}{2} \varphi(x)\left\langle D_{\xi} h, x^{2}\right\rangle=L \varphi(x)-\frac{1}{2}\left\langle D_{\xi} D \varphi(x), x^{2}\right\rangle,
$$

for any $x \in L^{6}(0,1)$. As easily seen, $\left|D_{\xi} e^{t A} h\right|_{2} \leq \pi\left|D_{\xi} h\right|_{2}$, then

$$
\left|\frac{P_{t} \varphi(x)-\varphi(x)}{t}\right| \leq\left|\frac{R_{t} \varphi(x)-\varphi(x)}{t}\right|+\frac{\left|D_{\xi} h\right|_{2}}{2 t} \int_{0}^{t} \mathbb{E}\left[|X(s, x)|_{4}^{2}\right] d s
$$

Now, since $\varphi \in D\left(L, \mathcal{C}_{b, 1}(H)\right)$, the first term of right-hand side is bounded by

$$
\left|\frac{R_{t} \varphi(x)-\varphi(x)}{t}\right| \leq c\left(1+|x|_{2}\right),
$$

where $c_{\varphi, T}>0$ depends only by $\varphi$ and T. By Proposition 1.1, the last term on the right-hand side is bounded by

$$
\frac{\left|D_{\xi} h\right|_{2}}{2 t} \int_{0}^{t} \mathbb{E}\left[|X(s, x)|_{4}^{2}\right] d s \leq \frac{\left|D_{\xi} h\right|_{2}}{2} \mathbb{E}\left[\sup _{t \in[0, T]}|X(t, x)|_{4}^{2}\right] d s \leq \frac{c_{T}\left|D_{\xi} h\right|_{2}}{2}\left(1+|x|_{4}^{2}\right),
$$


where $c_{T}>0$ depends only by $T$. Then,

$$
\sup _{t \in(0,1)}\left\|\frac{P_{t} \varphi-\varphi}{t}\right\|_{0, V}<\infty .
$$

This implies $\varphi \in D\left(K, \mathcal{C}_{b, V}\left(L^{6}(0,1)\right)\right)$ and $K \varphi=L_{V} \varphi-\frac{1}{2}\left\langle D_{\xi} D \varphi,(\cdot)^{2}\right\rangle$. Consequently, the claim follows by Proposition 5.5 .

Lemma 7.3. $\mathcal{E}_{A}(H)$ is a $\pi$-core for $\left(K, D\left(K, \mathcal{C}_{b, V}\left(L^{6}(0,1)\right)\right)\right)$, that is for any $\varphi \in D\left(K, \mathcal{C}_{b, V}\left(L^{6}(0,1)\right)\right)$ there exist $m \in \mathbb{N}$ and an $m$-indexed sequence $\left(\varphi_{n_{1}, \ldots, n_{m}}\right)_{n_{1} \in \mathbb{N}, \ldots, n_{m} \in \mathbb{N}} \subset \mathcal{E}_{A}(H)$ such that

$$
\lim _{n_{1} \rightarrow \infty} \cdots \lim _{n_{m} \rightarrow \infty} \frac{\varphi_{n_{1}, \ldots, n_{m}}}{1+V} \stackrel{\pi}{=} \frac{\varphi}{1+V}
$$

and

$$
\lim _{n_{1} \rightarrow \infty} \cdots \lim _{n_{m} \rightarrow \infty} \frac{K_{0} \varphi_{n_{1}, \ldots, n_{m}}}{1+V} \stackrel{\pi}{=} \frac{K \varphi}{1+V} .
$$

Step 1. Take $\varphi \in D\left(K, \mathcal{C}_{b, V}\left(L^{6}(0,1)\right)\right)$ and fix $\lambda>\omega_{0}, \omega_{1}$, where $\omega_{0}$ is as in Proposition 4.2 and $\omega_{1}$ is as in Proposition 6.2. We set $\lambda \varphi-K \varphi=f$. By Proposition 4.3 we have $\varphi=R(\lambda, K) f$. Let us fix a sequence $\left(f_{n_{1}}\right)_{n_{1} \in \mathbb{N}} \subset$ $\mathcal{E}_{A}(H)$ such that

$$
\lim _{n_{1} \rightarrow \infty} \frac{f_{n_{1}}}{1+V} \stackrel{\pi}{=} \frac{f}{1+V}
$$

We set $\varphi_{n_{1}}=R(\lambda, K) f_{n_{1}}$. By Proposition 4.2, 4.3 it follows

$$
\lim _{n_{1} \rightarrow \infty} \frac{\varphi_{n_{1}}}{1+V} \stackrel{\pi}{=} \frac{\varphi}{1+V}, \quad \lim _{n_{1} \rightarrow \infty} \frac{K \varphi_{n_{1}}}{1+V} \stackrel{\pi}{=} \frac{K \varphi}{1+V} .
$$

Step 2. Now let $\left(K_{m}, D\left(K_{m}, \mathcal{C}_{b, V}\left(L^{6}(0,1)\right)\right)\right)$ be the infinitesimal generator of the semigroup $P_{t}^{m}, t \geq 0$ in the space $\mathcal{C}_{b, V}\left(L^{6}(0,1)\right)$, as defined in (30). We set

$$
\varphi_{n_{1}, n_{2}}=\int_{0}^{\infty} e^{-\lambda t} P_{t}^{n_{2}} f_{n_{1}} d t
$$

By Proposition 4.3 we have $\varphi_{n_{1}, n_{2}} \in D\left(K_{n_{2}}, \mathcal{C}_{b, V}\left(L^{6}(0,1)\right)\right)$ and by a standard computation

$$
\lim _{n_{2} \rightarrow \infty} \frac{\varphi_{n_{1}, n_{2}}}{1+V} \stackrel{\pi}{=} \frac{\varphi_{n_{1}}}{1+V}, \quad \lim _{n_{2} \rightarrow \infty} \frac{K_{n_{2}} \varphi_{n_{1}, n_{2}}}{1+V} \stackrel{\pi}{=} \frac{K \varphi_{n_{1}}}{1+V} .
$$

Notice that $f_{n_{1}}$ satisfies the hypothesis of Proposition 6.3. Hence, $\varphi_{n_{1}, n_{2}} \in$ $D\left(L_{V}, \mathcal{C}_{b, V}\left(L^{6}(0,1)\right)\right)$ and

$$
K_{n_{2}} \varphi_{n_{1}, n_{2}}=L \varphi_{n_{1}, n_{2}}-\frac{1}{2}\left\langle D_{\xi} P_{n_{2}} D \varphi_{n_{1}, n_{2}},\left(P_{n_{2}} \cdot\right)^{2}\right\rangle
$$


for any $n_{1}, n_{2} \in \mathbb{N}, x \in L^{6}(0,1)$. In addition, by (31) it holds

$$
\begin{aligned}
& \left|\left\langle D_{\xi} D \varphi_{n_{1}, n_{2}}(x), x^{2}\right\rangle-\left\langle D_{\xi} P_{n_{2}} D \varphi_{n_{1}, n_{2}}(x),\left(P_{n_{2}} x\right)^{2}\right\rangle\right| \\
& =\left|\left\langle D \varphi_{n_{1}, n_{2}}(x), D_{\xi}\left(x^{2}\right)-P_{n_{2}} D_{\xi}\left(P_{n_{2}} x\right)^{2}\right\rangle\right| \\
& \leq\left|D \varphi_{n_{1}, n_{2}}(x)\right|_{H^{1}(0,1)}\left|D_{\xi}\left(x^{2}\right)-P_{n_{2}} D_{\xi}\left(P_{n_{2}} x\right)^{2}\right|_{W^{-1,2}(0,1)} \\
& \leq\left(\frac{\left\|D \varphi_{n_{1}}\right\|_{C_{b}\left(H ; H^{1}(0,1)\right)}+c\left\|\varphi_{n_{1}}\right\|_{0}}{\lambda-\omega_{1}}\right)\left(1+|x|_{6}\right)^{8} \\
& \quad \times\left|D_{\xi}\left(x^{2}\right)-P_{n_{2}} D_{\xi}\left(P_{n_{2}} x\right)^{2}\right|_{W^{-1,2}(0,1)}
\end{aligned}
$$

for any $x \in L^{6}(0,1)$, where $W^{-1,2}(0,1)$ is the topological dual of $H^{1}(0,1)$ endowed with the norm $|\cdot|_{W^{-1,2}(0,1)}$. Consequently,

$$
\lim _{n_{2} \rightarrow \infty} \frac{\left\langle D_{\xi} D \varphi_{n_{1}, n_{2}}(x), x^{2}\right\rangle-\left\langle D_{\xi} P_{n_{2}} D \varphi_{n_{1}, n_{2}}(x),\left(P_{n_{2}} x\right)^{2}\right\rangle}{1+V} \stackrel{\pi}{=} 0
$$

Step 3. By Proposition 6.4 for any $n_{1}, n_{2} \in \mathbb{N}$ there exists a sequence (we assume that it has one index) $\left\{\varphi_{n_{1}, n_{2}, n_{3}}\right\}_{n_{3} \in \mathbb{N}} \subset \mathcal{E}_{A}(H)$ such that

$$
\begin{gathered}
\lim _{n_{3} \rightarrow \infty} \frac{\varphi_{n_{1}, n_{2}, n_{3}}}{1+V} \stackrel{\pi}{=} \frac{\varphi_{n_{1}, n_{2}}}{1+V} \\
\lim _{n_{3} \rightarrow \infty} \frac{L_{0} \varphi_{n_{1}, n_{2}, n_{3}}}{1+V} \stackrel{\pi}{=} \frac{L \varphi_{n_{1}, n_{2}}}{1+V}
\end{gathered}
$$

and

$$
\lim _{n_{3} \rightarrow \infty} \frac{\left\langle D_{\xi} D \varphi_{n_{1}, n_{2}, n_{3}}, h\right\rangle}{1+|\cdot|_{6}^{8}} \stackrel{\pi}{=} \frac{\left\langle D_{\xi} D \varphi_{n_{1}, n_{2}}, h\right\rangle}{1+|\cdot|_{6}^{8}}, \quad \forall h \in H
$$

Then it follows

$$
\lim _{n_{3} \rightarrow \infty} \frac{\left\langle D_{\xi} P_{n_{2}} D \varphi_{n_{1}, n_{2}, n_{3}},(\cdot)^{2}\right\rangle}{1+V} \stackrel{\pi}{=} \frac{\left\langle D_{\xi} P_{n_{2}} D \varphi_{n_{1}, n_{2}},(\cdot)^{2}\right\rangle}{1+V} .
$$

Step 4. By construction, $\left(\varphi_{n_{1}, n_{2}, n_{3}}\right)_{n_{1}, n_{2}, n_{3}} \subset \mathcal{E}_{A}(H)$. By (51), (52), (55)

$$
\lim _{n_{1} \rightarrow \infty} \lim _{n_{2} \rightarrow \infty} \lim _{n_{3} \rightarrow \infty} \frac{\varphi_{n_{1}, n_{2}, n_{3}}}{1+V} \stackrel{\pi}{=} \frac{\varphi}{1+V} .
$$

Hence (49) follows. Let us show (50). By Lemma 7.2, for any $n_{1}, n_{2}, n_{3} \in \mathbb{N}$, $x \in L^{6}(0,1)$ we have

$$
K \varphi_{n_{1}, n_{2}, n_{3}}(x)=K_{0} \varphi_{n_{1}, n_{2}, n_{3}}(x)=L_{0} \varphi_{n_{1}, n_{2}, n_{3}}(x)-\frac{1}{2}\left\langle D_{\xi} \varphi_{n_{1}, n_{2}, n_{3}}(x), x^{2}\right\rangle .
$$


By (56), (157),

$$
\lim _{n_{3} \rightarrow \infty} \frac{K_{0} \varphi_{n_{1}, n_{2}, n_{3}}}{1+V} \stackrel{\pi}{=} \frac{L \varphi_{n_{1}, n_{2}}-\frac{1}{2}\left\langle D_{\xi} D \varphi_{n_{1}, n_{2}},(\cdot)^{2}\right\rangle}{1+V}
$$

By (53) it holds

$$
\begin{aligned}
L \varphi_{n_{1}, n_{2}} & -\frac{1}{2}\left\langle D_{\xi} D \varphi_{n_{1}, n_{2}},(\cdot)^{2}\right\rangle \\
& =K_{n_{2}} D \varphi_{n_{1}, n_{2}}+\frac{1}{2}\left\langle D_{\xi} P_{n_{2}} D \varphi_{n_{1}, n_{2}},\left(P_{n_{2}} \cdot\right)^{2}\right\rangle-\frac{1}{2}\left\langle D_{\xi} D \varphi_{n_{1}, n_{2}},(\cdot)^{2}\right\rangle
\end{aligned}
$$

By (152), (154)

$$
\lim _{n_{3} \rightarrow \infty} \frac{L \varphi_{n_{1}, n_{2}, n_{3}}-\frac{1}{2}\left\langle D_{\xi} D \varphi_{n_{1}, n_{2}, n_{3}},(\cdot)^{2}\right\rangle}{1+V} \stackrel{\pi}{=} \frac{K \varphi_{n_{1}, n_{2}}}{1+V}
$$

Finally, by (151), (152) we have

$$
\lim _{n_{1} \rightarrow \infty} \lim _{n_{2} \rightarrow \infty} \frac{K \varphi_{n_{1}, n_{2}}}{1+V} \stackrel{\pi}{=} \frac{K \varphi}{1+V}
$$

\section{The Fokker-Planck equation and Proof of Theorem 1.2}

Since the semigroup $P_{t}, t \geq 0$ is markovian and Theorem 3.1 holds, it follows that $P_{t}, t \geq 0$ acts on $\mathcal{C}_{b}\left(L^{6}(0,1)\right)$. In particular, we have that $P_{t}, t \geq 0$ is a $\pi$-semigroup on $\mathcal{C}_{b}\left(L^{6}(0,1)\right)$, as introduced in [24]24, 24]. We define its infinitesimal generator by

$$
\left\{\begin{array}{c}
D\left(K, \mathcal{C}_{b}\left(L^{6}(0,1)\right)\right)=\left\{\varphi \in D\left(K, \mathcal{C}_{b}\left(L^{6}(0,1)\right)\right): \exists g \in \mathcal{C}_{b}\left(L^{6}(0,1)\right),\right. \\
\left.\lim _{t \rightarrow 0^{+}} \frac{P_{t} \varphi(x)-\varphi(x)}{t}=g(x), x \in L^{6}(0,1), \sup _{t \in(0,1)}\left\|\frac{P_{t} \varphi-\varphi}{t}\right\|_{0}<\infty\right\} \\
K \varphi(x)=\lim _{t \rightarrow 0^{+}} \frac{P_{t} \varphi(x)-\varphi(x)}{t}, \quad \varphi \in D\left(K, \mathcal{C}_{b}\left(L^{6}(0,1)\right)\right), x \in L^{6}(0,1) .
\end{array}\right.
$$

We have the following

Theorem 8.1. The family of linear maps $P_{t}^{*}:\left(\mathcal{C}_{b}\left(L^{6}(0,1)\right)\right)^{*} \rightarrow\left(\mathcal{C}_{b}\left(L^{6}(0,1)\right)\right)^{*}$, $t \geq 0$, defined by the formula

$$
\left\langle\varphi, P_{t}^{*} F\right\rangle_{\sigma\left(\mathcal{C}_{b}\left(L^{6}(0,1)\right),\left(\mathcal{C}_{b}\left(L^{6}(0,1)\right)\right)^{*}\right)}=\left\langle P_{t} \varphi, F\right\rangle_{\sigma\left(\mathcal{C}_{b}\left(L^{6}(0,1)\right),\left(\mathcal{C}_{b}\left(L^{6}(0,1)\right)\right)^{*}\right)},
$$


where $t \geq 0, F \in\left(\mathcal{C}_{b}\left(L^{6}(0,1)\right)\right)^{*}, \varphi \in \mathcal{C}_{b}\left(L^{6}(0,1)\right)$, is a semigroup of linear operators on $\left(\mathcal{C}_{b}\left(L^{6}(0,1)\right)\right)^{*}$ which is stable on $\mathcal{M}\left(L^{6}(0,1)\right)$. Moreover, for any $\mu \in \mathcal{M}\left(L^{6}(0,1)\right)$ there exists a unique family of measures $\left\{\mu_{t}, t \geq 0\right\} \subset$ $\mathcal{M}\left(L^{6}(0,1)\right)$ fulfilling

$$
\begin{gathered}
\int_{0}^{T}\left|\mu_{t}\right|_{T V}\left(L^{6}(0,1)\right) d t<\infty, \quad T>0 \\
\int_{L^{6}(0,1)} \varphi(x) \mu_{t}(d x)-\int_{L^{6}(0,1)} \varphi(x) \mu(d x) \\
=\int_{0}^{t}\left(\int_{L^{6}(0,1)} K \varphi(x) \mu_{s}(d x)\right) d s,
\end{gathered}
$$

for any $\varphi \in D\left(K, \mathcal{C}_{b}\left(L^{6}(0,1)\right)\right), t \geq 0$. Finally, the solution of (59), (60) is given by $P_{t}^{*} \mu, t \geq 0$.

Proof. The proof of this theorem is very similar to the proof of Theorem 1.2 of $[20 \mid 20,20]$. We stress that in [20120, 20] the space $L^{6}(0,1)$ is replaced by a separable Hilbert space $H$ and that $\mathcal{C}_{b}\left(L^{6}(0,1)\right)$ is replaced by the space $C_{b}(H)$, the Banach space of all the uniformly continuous and bounded functions $\varphi: H \rightarrow \mathbb{R}$, endowed with the supremum norm. However, one can see that all the results remain true with continuity replacing uniform continuity and $L^{6}(0,1)$ replacing $H$ (see, also, [23[23, 23]).

Thanks to Propositions 4.2 and 4.3, the above theorem can be extended to the space $\mathcal{C}_{b, V}\left(L^{6}(0,1)\right)$.

Theorem 8.2. Let $\left(P_{t}\right)_{t \geq 0}$ be the semigroup defined by (3) and let us consider its infinitesimal generator $\left(K, D\left(K, \mathcal{C}_{b, V}\left(L^{6}(0,1)\right)\right)\right)$ given by (4). Then, the formula

$$
\left\langle\varphi, P_{t}^{*} F\right\rangle_{\sigma\left(\mathcal{C}_{b, V}\left(L^{6}(0,1)\right),\left(\mathcal{C}_{b, V}\left(L^{6}(0,1)\right)\right)^{*}\right)}=\left\langle P_{t} \varphi, F\right\rangle_{\sigma\left(\mathcal{C}_{b, V}\left(L^{6}(0,1)\right),\left(\mathcal{C}_{b, V}\left(L^{6}(0,1)\right)\right)^{*}\right)}
$$

defines a semigroup $P_{t}^{*}, t \geq 0$ of linear and continuous operators on $\left(\mathcal{C}_{b, V}\left(L^{6}(0,1)\right)^{*}\right.$ which is stable on $\mathcal{M}_{V}\left(L^{6}(0,1)\right)$. Moreover, for any measure $\mu \in \mathcal{M}_{V}\left(L^{6}(0,1)\right)$ there exists a unique family $\left\{\mu_{t}, t \geq 0\right\} \subset \mathcal{M}_{V}\left(L^{6}(0,1)\right)$ such that (66) holds and

$$
\begin{aligned}
\int_{L^{6}(0,1)} \varphi(x) \mu_{t}(d x)-\int_{L^{6}(0,1)} \varphi(x) \mu & (d x) \\
& =\int_{0}^{t}\left(\int_{L^{6}(0,1)} K \varphi(x) \mu_{s}(d x)\right) d s
\end{aligned}
$$

for any $t \geq 0, \varphi \in D\left(K, \mathcal{C}_{b, V}\left(L^{6}(0,1)\right)\right)$. Finally, the solution of (66), (61) is given by $P_{t}^{*} \mu, t \geq 0$. 
Proof. Since $P_{t}, t \geq 0$ acts on $\mathcal{C}_{b, V}\left(L^{6}(0,1)\right)$ (see Proposition 4.2), it follows easily that $P_{t}^{*}$ acts on $\left(\mathcal{C}_{b, V}\left(L^{6}(0,1)\right)\right)^{*}$. Let us show that $P_{t}^{*}$ is stable on $\mathcal{M}_{V}\left(L^{6}(0,1)\right)$. Take $\mu \in \mathcal{M}_{V}\left(L^{6}(0,1)\right)$. By the linearity of $P_{t}^{*}$, it is sufficient to take $\mu$ non negative. By Theorem 8.1 we have $P_{t}^{*} \mu \in \mathcal{M}\left(L^{6}(0,1)\right)$. Consider a sequence of functions $\left(\psi_{n}\right)_{n \in \mathbb{N}} \subset C_{b}\left(L^{6}(0,1)\right)$ such that $\psi_{n}(x) \rightarrow V(x)$ as $n \rightarrow \infty$ and $0 \leq \psi(x) \leq V(x)$, for any $x \in L^{6}(0,1)$. By Proposition 4.2 we have that

$$
\begin{aligned}
\lim _{n \rightarrow \infty} P_{t} \psi_{n}(x) & =\lim _{n \rightarrow \infty} \int_{L^{6}(0,1)} \psi_{n}(y) \pi_{t}(x, d y) \\
& =\int_{L^{6}(0,1)} V(y) \pi_{t}(x, d y)=P_{t} V(x)
\end{aligned}
$$

and

$$
P_{t} \psi_{n}(x)=\int_{L^{6}(0,1)} \psi_{n}(y) \pi_{t}(x, d y) \leq \int_{L^{6}(0,1)} V(y) \pi_{t}(x, d y) \leq c(1+V(x))
$$

hold for any $x \in L^{6}(0,1)$ and for some $c>0$. Hence, since $\mu \in \mathcal{M}_{V}\left(L^{6}(0,1)\right)$ by the dominated convergence Theorem we have

$$
\begin{aligned}
\int_{L^{6}(0,1)} V(x) P_{t}^{*} \mu(d x) & =\lim _{n \rightarrow \infty} \int_{L^{6}(0,1)} \psi_{n}(x) P_{t}^{*} \mu(d x) \\
& =\lim _{n \rightarrow \infty} \int_{L^{6}(0,1)} P_{t} \psi_{n}(x) \mu(d x) \\
& =\lim _{n \rightarrow \infty} \int_{L^{6}(0,1)}\left(\int_{L^{6}(0,1)} \psi_{n}(y) \pi_{t}(x, d y)\right) \mu(d x) \\
& =\int_{L^{6}(0,1)} P_{t} V(x) \mu(d x) .
\end{aligned}
$$

Then, by (62)

$$
\int_{L^{6}(0,1)} V(x) P_{t}^{*} \mu(d x) \leq \int_{L^{6}(0,1)} \leq c(1+V(x)) \mu(d x)<\infty,
$$

since $\mu \in \mathcal{M}_{V}\left(L^{6}(0,1)\right)$. Recalling that $P_{t}^{*} \mu \in \mathcal{M}\left(L^{6}(0,1)\right)$, it follows $P_{t}^{*} \mu \in$ $\mathcal{M}_{V}\left(L^{6}(0,1)\right)$.

Let us now prove the remaining part of the theorem. We split the proof in two steps.

Step 1: Existence of a solution of (6) , (61). Let us fix $\mu \in \mathcal{M}_{V}\left(L^{6}(0,1)\right)$. By the first part of the Theorem, we have $P_{s}^{*} \mu \in \mathcal{M}_{V}\left(L^{6}(0,1)\right)$, for any $s \geq 0$. We now show that for any $\varphi \in D\left(K, \mathcal{C}_{b, V}\left(L^{6}(0,1)\right)\right)$ the function

$$
[0, \infty) \rightarrow \mathbb{R}, \quad s \mapsto \int_{L^{6}(0,1)} P_{s} \varphi(x) \mu(d x)
$$


is differentiable, with continuous differential

$$
t \mapsto \int_{L^{6}(0,1)} K \varphi(x) P_{s}^{*} \mu(d x)
$$

By (44) we have, for any $\varphi \in D\left(K, \mathcal{C}_{b, V}\left(L^{6}(0,1)\right)\right)$,

$$
\begin{aligned}
\lim _{h \rightarrow 0^{+}} & \frac{1}{h}\left(\int_{L^{6}(0,1)} P_{s+h} \varphi(x) \mu(d x)-\int_{L^{6}(0,1)} P_{s} \varphi(x) \mu(d x)\right) \\
& =\lim _{h \rightarrow 0^{+}} \frac{1}{h}\left(\int_{L^{6}(0,1)} K P_{h} \varphi(x) P_{s}^{*} \mu(d x)-\int_{L^{6}(0,1)} \varphi(x) P_{s}^{*} \mu(d x)\right) \\
& =\lim _{h \rightarrow 0^{+}} \int_{L^{6}(0,1)} \frac{P_{h} \varphi(x)-\varphi(x)}{h} P_{s}^{*} \mu(d x) \\
& =\int_{L^{6}(0,1)} K \varphi(x) P_{s}^{*} \mu(d x)
\end{aligned}
$$

Since $K \varphi \in \mathcal{C}_{b, V}\left(L^{6}(0,1)\right)$, we have

$$
\int_{L^{6}(0,1)} K \varphi(x) P_{s}^{*} \mu(d x)=\int_{L^{6}(0,1)} P_{s} K \varphi(x) \mu(d x) .
$$

Then, by Proposition 4.3, this is a continuous as function of $s$. By the fundamental theorem of calculus it follows that $P_{t}^{*} \mu, t \geq 0$ solves (6), (61).

Uniqueness of a solution. Assume that $\left\{\mu_{t}, t \geq 0\right\} \subset \mathcal{M}_{V}\left(L^{6}(0,1)\right)$ fulfills (6), (61). It is straightforward to show that $D\left(K, C_{b}\left(L^{6}(0,1)\right)\right) \subset$ $D\left(K, C_{b, V}\left(L^{6}(0,1)\right)\right)$. Then (61) holds for any $\varphi \in D\left(K, C_{b}\left(L^{6}(0,1)\right)\right)$. It is also obvious that (6) implies (59). Then, by Theorem 8.1, if follows that $\mu_{t}=P_{t}^{*} \mu$, for any $t \geq 0$.

\subsection{Proof of Theorem 1.2}

Take $\mu \in \mathcal{M}_{V}\left(L^{6}(0,1)\right)$. As before, we split the proof in two steps.

Existence of a solution of (6) , (7). Fix $\mu \in \mathcal{M}_{V}\left(L^{6}(0,1)\right)$. By Theorem 8.2 we have that the family $P_{t}^{*} \mu, t \geq 0$ fulfills (6) . On the other hand, by Theorem 7.1 we have $\mathcal{E}_{A}(H) \subset D\left(K, \mathcal{C}_{b, V}\left(L^{6}(0,1)\right)\right)$ and $K \varphi=K_{0} \varphi$ for any $\varphi \in \mathcal{E}_{A}(H)$. Then, by (61) it follows that (7) holds for any $t \geq 0, \varphi \in \mathcal{E}_{A}(H)$. Then, $P_{t}^{*} \mu, t \geq 0$ is solution of (66), (7).

Uniqueness of the solution. Assume that $\left\{\mu_{t}, t \geq 0\right\} \subset \mathcal{M}_{V}\left(L^{6}(0,1)\right)$ fulfills (66), (7). Take $\varphi \in \mathcal{C}_{b, V}\left(L^{6}(0,1)\right)$. By Theorem 7.1 there exist $m \in \mathbb{N}$ and an $m$-indexed sequence $\left(\varphi_{n_{1}, \ldots, n_{m}}\right)_{n_{1}, \ldots, n_{m} \in \mathbb{N}} \subset \mathcal{E}_{A}(H)$ such that

$$
\lim _{n_{1} \rightarrow \infty} \cdots \lim _{n_{m} \rightarrow \infty} \frac{\varphi_{n_{1}, \ldots, n_{m}}}{1+V} \stackrel{\pi}{=} \frac{\varphi}{1+V}
$$


and

$$
\lim _{n_{1} \rightarrow \infty} \cdots \lim _{n_{m} \rightarrow \infty} \frac{K_{0} \varphi_{n_{1}, \ldots, n_{m}}}{1+V} \stackrel{\pi}{=} \frac{K \varphi}{1+V}
$$

Then, since $\left\{\mu_{t}, t \geq 0\right\} \subset \mathcal{M}_{V}\left(L^{6}(0,1)\right)$, by the dominated convergence theorem we have

$$
\begin{gathered}
\lim _{n_{1} \rightarrow \infty} \cdots \lim _{n_{m} \rightarrow \infty}\left(\int_{L^{6}(0,1)} \varphi_{n_{1}, \ldots, n_{m}}(x) \mu_{t}(d x)-\int_{L^{6}(0,1)} \varphi_{n_{1}, \ldots, n_{m}}(x) \mu(d x)\right) \\
=\int_{L^{6}(0,1)} \varphi(x) \mu_{t}(d x)-\int_{L^{6}(0,1)} \varphi(x) \mu(d x)
\end{gathered}
$$

Similarly, for any $s \in[0, t]$ we have

$$
\begin{gathered}
\lim _{n_{1} \rightarrow \infty} \cdots \lim _{n_{m} \rightarrow \infty} \int_{L^{6}(0,1)} K_{0} \varphi_{n_{1}, \ldots, n_{m}}(x) \mu_{s}(d x) \\
=\int_{L^{6}(0,1)} K \varphi(x) \mu_{s}(d x) .
\end{gathered}
$$

Therefore, by (66) we can still apply the dominated convergence theorem to find

$$
\begin{gathered}
\lim _{n_{1} \rightarrow \infty} \cdots \lim _{n_{m} \rightarrow \infty} \int_{0}^{t}\left(\int_{L^{6}(0,1)} K_{0} \varphi_{n_{1}, \ldots, n_{m}}(x) \mu_{s}(d x)\right) d s \\
=\int_{0}^{t}\left(\int_{L^{6}(0,1)} K \varphi(x) \mu_{s}(d x)\right) d s .
\end{gathered}
$$

Then, $\left\{\mu_{t}, t \geq 0\right\}$ is solution of (6) and (61) , for any $\varphi \in D\left(K, C_{b, V}\left(L^{6}(0,1)\right)\right)$. But by Theorem 8.2 such a solution is unique, thus $\mu_{t}$ must coincide with $P_{t}^{*} \mu, \forall t \geq 0$. The proof is complete.

\section{References}

[1] ]cite.BR011Vladimir I. Bogachev and Michael Röckner, Elliptic equations for measures on infinite-dimensional spaces and applications, Probab. Theory Related Fields 120 (2001), no. 4, 445-496.

[2] ]cite.Cerrai2Sandra Cerrai, A Hille-Yosida theorem for weakly continuous semigroups, Semigroup Forum 49 (1994), no. 3, 349-367.

[3] ]cite.DPDT3Giuseppe Da Prato, Arnaud Debussche, and Roger Temam, Stochastic Burgers' equation, NoDEA Nonlinear Differential Equations Appl. 1 (1994), no. 4, 389-402. 
[4] ]cite.DPD984Giuseppe Da Prato and Arnaud Debussche, Differentiability of the transition semigroup of the stochastic Burgers equation, and application to the corresponding Hamilton-Jacobi equation, Atti Accad. Naz. Lincei Cl. Sci. Fis. Mat. Natur. Rend. Lincei (9) Mat. Appl. 9 (1998), no. 4, 267-277 (1999).

[5] ]cite.DPD005_, Dynamic programming for the stochastic Burgers equation, Ann. Mat. Pura Appl. (4) 178 (2000), 143-174.

[6] ]cite.DPD00a6_, Dynamic programming for the stochastic Navier-Stokes equations, M2AN Math. Model. Numer. Anal. 34 (2000), no. 2, 459-475. Special issue for R. Temam's 60th birthday.

[7] ]cite.DPD037_, Ergodicity for the 3D stochastic Navier-Stokes equations, J. Math. Pures Appl. (9) 82 (2003), no. 8, 877-947.

[8] ]cite.DPD078___ m-dissipativity of Kolmogorov operators corresponding to Burgers equations with space-time white noise, Potential Anal. 26 (2007), no. 1, 31-55.

[9] ]cite.DPT019Giuseppe Da Prato and Luciano Tubaro, Some results about dissipativity of Kolmogorov operators, Czechoslovak Math. J. 51(126) (2001), no. 4, 685-699.

[10] ]cite.DPZ9210Giuseppe Da Prato and Jerzy Zabczyk, Stochastic equations in infinite dimensions, Encyclopedia of Mathematics and its Applications, vol. 44, Cambridge University Press, 1992.

[11] ]cite.DPZ9611_, Ergodicity for infinite-dimensional systems, London Mathematical Society Lecture Note Series, vol. 229, Cambridge University Press, 1996.

[12] ]cite.DPZ9712_, Differentiability of the Feynman-Kac semigroup and a control application, Atti Accad. Naz. Lincei Cl. Sci. Fis. Mat. Natur. Rend. Lincei (9) Mat. Appl. 8 (1997), no. 3, 183-188.

[13] ]cite.DPZ0213_ Second order partial differential equations in Hilbert spaces, London Mathematical Society Lecture Note Series, vol. 293, Cambridge University Press, 2002.

[14] ]cite.DP0414Giuseppe Da Prato, Kolmogorov equations for stochastic PDEs, Advanced Courses in Mathematics. CRM Barcelona, Birkhäuser Verlag, 2004.

[15] ]cite.DO15Arnaud Debussche and Cyril Odasso, Markov solutions for the 3D stochastic Navier-Stokes equations with state dependent noise, J. Evol. Equ. 6 (2006), no. 2, $305-324$.

[16] ]cite.GK0116B. Goldys and M. Kocan, Diffusion semigroups in spaces of continuous functions with mixed topology, J. Differential Equations 173 (2001), no. 1, 17-39.

[17] ]cite.Henry17Daniel Henry, Geometric theory of semilinear parabolic equations, Lecture Notes in Mathematics, vol. 840, Springer-Verlag, Berlin, 1981.

[18] ]cite.LaTh18T. Lant and H. R. Thieme, Markov transition functions and semigroups of measures, Semigroup Forum 74 (2007), no. 3, 337-369.

[19] ]cite.Manca0619Luigi Manca, On a class of stochastic semilinear PDEs, Stoch. Anal. Appl. 24 (2006), no. 2, 399-426.

[20] ]cite.Manca0720_ Kolmogorov equations for measures, 2007. To appear on Journal of Evolution Equations. 
[21] ]cite.Manca07a21 Measure-valued equations for Kolmogorov operators with unbounded coefficients, 2007. Preprint.

[22] ]cite.Mancacontrol0722 On dynamic programming approach for the $3 d$-NavierStokes equations, 2007. To be published on Appl. Math. Optim.

[23] ]cite.Manca0823 Kolmogorov operators in spaces of continuous functions and equations for measures, Ph.D. Thesis, 2008.

[24] ]cite.Priola24Enrico Priola, On a class of Markov type semigroups in spaces of uniformly continuous and bounded functions, Studia Math. 136 (1999), no. 3, 271-295.

[25] ]cite.RS0425Michael Röckner and Zeev Sobol, A new approach to Kolmogorov equations in infinite dimensions and applications to stochastic generalized Burgers equations, C. R. Math. Acad. Sci. Paris 338 (2004), no. 12, 945-949.

[26] ]cite.RS0626_ Kolmogorov equations in infinite dimensions: well-posedness and regularity of solutions, with applications to stochastic generalized Burgers equations, Ann. Probab. 34 (2006), no. 2, 663-727.

[27] ]cite.Rothe27Franz Rothe, Global solutions of reaction-diffusion systems, Lecture Notes in Mathematics, vol. 1072, Springer-Verlag, Berlin, 1984. 